Article

\title{
Scattering on Quasi-Spherical Black-Holes: Features and Beyond
}

\author{
Adam M. Arslanaliev ${ }^{1,2}$ and Alexei J. Nurmagambetov 1,2,3,*(D) \\ 1 Department of Physics and Technology, Karazin Kharkov National University, 4 Svobody Sq., \\ UA 61022 Kharkov, Ukraine; arslanaliev.kh@gmail.com \\ 2 Akhiezer Institute for Theoretical Physics of NSC KIPT, 1 Akademicheskaya St., UA 61108 Kharkov, Ukraine \\ 3 Usikov Institute for Radiophysics and Electronics, 12 Proskura St., UA 61085 Kharkov, Ukraine \\ * Correspondence: ajn@kipt.kharkov.ua
}

check for

updates

Citation: Arslanaliev, A.M.;

Nurmagambetov, A.J. Scattering on Quasi-Spherical Black-Holes:

Features and Beyond. Physics 2021, 3,

17-41. https://doi.org/10.3390/

physics3010004

Received: 30 November 2020

Accepted: 22 January 2021

Published: 28 January 2021

Publisher's Note: MDPI stays neutral with regard to jurisdictional clai$\mathrm{ms}$ in published maps and institutional affiliations.

Copyright: $(\odot 2021$ by the authors. Licensee MDPI, Basel, Switzerland. This article is an open access article distributed under the terms and conditions of the Creative Commons Attribution (CC BY) license (https:// creativecommons.org/licenses/by/ $4.0 /)$.

\begin{abstract}
Recent developments in the gravitational waves interferometry require more pertinent theoretical models of gravitational waves generation and propagation. Untouched possible mechanisms of spin-2 spacetime perturbations production, we will consider their subsequent scattering on other black holes (BHs). Specifically, we consider a generalization of the Regge-Wheeler-Zerilli equations for the case of distorted BHs (BHs surrounded with matter) in Minkowski and Anti-de Sitter spacetimes, the metric potential of which obeys the Liouville equation. We establish significant differences in scattering characteristics of waves of different spins and angular momenta, including the gravitational waves, caused by losing the spherical symmetry of their propagation background. In particular, we demonstrate the strong impact of the background geometry deformation on the grey-body factors, hence on the absorption cross-sections of scattering waves, and explore the issue of stability of the background geometry upon changing the deformation degree parameters.
\end{abstract}

Keywords: black holes; Regge-Wheeler-Zerilli equations; quasinormal modes; gravitational waves

\section{Introduction}

Progress in observation astronomy is going so fast that we become beholders of transforming astrophysics into genuinely physical discipline, where theoretical predictions are supervised with experimentally verified data. A lot of discoveries in the field were done since the century beginning. We will outline just a few breakthroughs in the contemporary multi-messenger astrophysics [1], related in different ways to black holes (BHs):

- $\quad$ First, we have the LIGO-Virgo scientific collaborations to mention for their fundamental contribution in registrations of gravitational waves (GWs) [2]. It is believed that the GWs are mostly induced in processes which involve BHs.

- Second, it is important to notice the detection of ultra high energy cosmic rays (UHECRs) of energy $\sim 10^{19} \mathrm{eV}$-that far exceeds energies of the LHC (Large Hadron Collider)—coming from active galactic nuclei (AGN) [3]. It is believed that supermassive BHs (SMBHs) are located in the core of the AGN [4,5], and mechanisms of the UHECRs generation are directly related to BH physics; see, e.g., Refs. [6-19], in this respect.

- $\quad$ Next, it is worth mentioning achievements of the Event Horizon Telescope (EHT) collaboration in revealing the $\mathrm{BH}$ event horizon (for $\mathrm{M} 87 \mathrm{SMBH}$ with the mass $\left.6.5 \times 10^{9} M_{\odot}[20]\right)$. Due to the EHT activity it is believed more and more that Astrophysical BHs (BHs in the sky) share common properties with mathematical BHs (solutions to Einstein equations).

- Last but not least: the recent data analysis of the EHT team puts the Einstein theory on the test [21]. It turns out that general relativity works well, and many alternative theories of gravity should be discarded as that of contradicting experimental data. 
However, one has to notice that the conclusions of Ref. [21] cannot be considered as ultimate ones; see, e.g., Ref. [22], in this respect.

The mentioned achievements in contemporary astrophysics show how various studies of compact astrophysical objects give new prospects in resolving old and revealing new problems, which impact the development of physics at whole.

A large enough amount of information on BHs comes with d-waves (spin 2 waves; GWs). In view of this fact, any current studies of d-waves become potentially important. One of the branches in this activity is scattering d-waves on compact astrophysical objects. Starting from first seminal papers on scattering of different spin particles on Kerr and Schwarzschild BHs [23-29], it has been obtained, analytically and numerically, crosssections and grey-body factors of external particle fluxes propagating in effective potentials of various BH models; see, e.g., Refs. [30-43].

In what follows, we will be interested in solving to the scattering problem for the GWs in the background of the so-called distorted BHs. Rationales behind appearing the distorted $\mathrm{BHs}$ on the scene come from the point that the Schwarzschild solution is highly idealized and cannot be fully employed in any real astrophysical problem; the same concerns the Kerr solution. Any presence of matter does not just change the metric, but may drastically change its type, due to the backreaction of matter fields enforcing the spacetime geometry to evolve in time. However, time-dependent solutions to the Einstein equations in analytical form are extremely rare (and for very specific matter fields) [44,45] and generally require involved numerical simulations. A significant simplification occurs upon replacing the backreaction of matter with an effective distortion of a static/stationary BH metric with keeping the time-independence of the solution. Then, the solution describing a static distribution of matter localized outside the $\mathrm{BH}$ horizon and the vacuum spacetime at its proximity is called the distorted BH [46,47]; also see Refs. [48,49]. There are various astrophysical applications for the distorted BH-type metrics (see, e.g., Refs. [50-57]), among which one can find the effective description of a double (neutron) "star" system, when the tidal forces of one of the components deforming the shape of the other are mimicked in the solution [58-60].

In the next parts of the paper, we will explore the scattering of $d$-waves on another class of distorted BHs, in which spacetime configuration is defined by a specific solution originally proposed and studied in Refs. [61-64] and later rediscovered in Ref. [65] in a different context. In Section 2, we briefly discuss the relationship between the standard metric of axisymmetric distorted $\mathrm{BH}[46,47]$ and the quasi-spherical BH solution of Refs. [61-64]. In its customary formulation, the Weyl solution includes two metric potentials (functions of the radial direction and polar angle) and describes a family of distorted BHs in dependence on their specific choice. The proposed generalization of the Weyl solution with third metric potential, in Section 2, now depending on two angles, breaks the original axial symmetry. It results in fixing two metric potentials of the Weyl solution, so that the final geometry becomes that of a quasi-spherical $\mathrm{BH}$, the metric potential of which obeys the Liouville equation. The quasi-spherical solution also describes a family of BHs, now determined by the choice of a single metric potential. The local spacetime geometry we will explore further on is that of a static neutral distorted BH. It is evidently not enough to describe real astrophysical problems in full extent (see more on a quasi-spherical generalization of the Kerr solution in the last section). However, it is sufficient to reveal main features in the scattering processes of external particle fluxes, which will also be inherent in scattering by rotating distorted black holes.

In Section 3, we consider equations for gravitational perturbations over backgrounds of quasi-spherical neutral BHs in Minkowski and anti-de Sitter (AdS) spacetimes and examine the issue of separation of variables. As it is well known, a possibility to separate the variables in dynamical equations of relativistic fields in a curved background is related to symmetry properties of the background spacetime. By use of the Newman-Penrose formalism, we figure out the spacetime of quasi-spherical BHs is of type D in the Petrov classification. Therefore, the radial and angular parts of equations for small perturbations 
over the quasi-spherical BH background can be set apart, and the resulting expressions generalize the Regge-Wheeler-Zerilli equations and the spherical harmonics equation.

In Section 4, we focus on the angular part of the scattering problem and survey differences in solution to the angular differential equation for the distorted/quasi-spherical spacetime in compare to the standard Schwarzschild background. In general, the solution to the angular equation in a quasi-spherical BH background is reduced to the spectral problem for infinite-dimensional matrices. For the background with axial symmetry, viable in many astrophysical problems, we can make further simplifications, and to solve the spectral problem numerically. Here, we find the principle difference between the quasi-spherical and spherically-symmetric cases, which will have the foremost impact on the scattering process: the eigenvalues of the examined spectral problem are not integers anymore; for each scattering mode, there is a set of eigenvalues, the number of which is determined by the corresponding to spherical symmetry value of the scattering mode angular momentum and by its projections (i.e., by the set of $(l, m)$ ); and finally, the eigenvalues depend on the deformation degree of the background geometry from spherically-symmetric, specified by a single parameter. On account of numerical computations we recovered the functional dependence of the generalized eigenvalues on the degree of deformation in the axiallysymmetric case.

Section 5 contains computations of the grey-body factor for different scattering waves on a quasi-spherical Schwarzschild BH and further comparison of the regular sphericallysymmetric case [43] to its counter-part with a non-trivial deformation. Because the generalized eigenvalues found in the preceding section carry on two indices and enter the Regge-Wheeler-Zerilli equations via the separation constant, numerically computed greybody factors for each type of perturbations-scalar, vector, and tensor-also become differentiated by $(l, m)$ indices. Explicitly, for every scattering mode with the angular momentum $l$, we find $l+1$ different values of the grey-body factor, properties of which were compared to that of the spherically-symmetric case. In particular, we find that the grey-body factors, as functions of the deformation degree, increase with increasing the value of this parameter, as well as that the transparency of the effective potential is reached for $(l, l)$ scattering modes at the lowest value of corresponding frequencies.

In Section 6, we consider the issue of stability of $\mathrm{BH}$ backgrounds by studying the quasinormal modes (QNMs). Here, we briefly review the relation between the stability of a spacetime and positivity of effective potentials in the Regge-Wheeler-Zerilli equations. Since the effective potential of small perturbations over distorted/quasi-spherical BH backgrounds depends on the deformation degree, this parameter becomes crucial for determining the stability of the background geometry against small perturbations. We find that the value of the deformation degree equal to one is the critical value for the stability of an axially-symmetric quasi-spherical Schwarzschild BH in Minkowski and AdS spacetimes. And, if the result does not depend on the size of a BH in flat spacetime, the instability of a Schwarzschild-AdS BH may only be encountered for the so-called large BHs, the event horizons of which are of the next order in compare to the characteristic scale of empty AdS space.

Finally, discussion of the results and summary of our findings are collected in the last section.

\section{Background Metric: From Distorted to Quasi-Spherical Black Hole}

Let us begin with a clarification of how the background metric, mainly used throughout the paper, is related to the metric of a distorted BH.

A distorted $\mathrm{BH}$ solution to the flat spacetime Einstein vacuum equations is traditionally described by the Weyl axisymmetric metric [46] in the cylindrical space-time coordinates $(t, \rho, \theta, \varphi)$ [47], $t$ is the time coordinate. However, when the case is about a static axisymmetric solution with an arbitrary quadrupole moment, things get essentially simplified in the prolate spheroidal space-time coordinates $(t, x, y, \varphi)[66]$, in which the line element looks as follows: 


$$
\begin{gathered}
d s^{2}=-e^{2 \psi(x, y)} d t^{2}+M^{2} e^{-2 \psi(x, y)}\left[e^{2 \gamma(x, y)}\left(x^{2}-y^{2}\right)\left(\frac{d x^{2}}{x^{2}-1}+\frac{d y^{2}}{1-y^{2}}\right)+\left(x^{2}-1\right)\left(1-y^{2}\right) d \varphi^{2}\right] . \\
\text { To obey the Einstein equations in vacuum, the metric potentials } \psi(x, y) \text { and } \gamma(x, y) \text { of } \\
\text { (1) should fulfill the following set of equations [66,67]: } \\
\qquad \partial_{x}\left(\left(x^{2}-1\right) \partial_{x} \psi\right)+\partial_{y}\left(\left(1-y^{2}\right) \partial_{y} \psi\right)=0, \\
\partial_{x} \gamma=\frac{1-y^{2}}{x^{2}-y^{2}}\left[x\left(x^{2}-1\right)\left(\partial_{x} \psi\right)^{2}-x\left(1-y^{2}\right)\left(\partial_{y} \psi\right)^{2}-2 y\left(x^{2}-1\right) \partial_{x} \psi \partial_{y} \psi\right] \\
\partial_{y} \gamma=\frac{x^{2}-1}{x^{2}-y^{2}}\left[y\left(x^{2}-1\right)\left(\partial_{x} \psi\right)^{2}-y\left(1-y^{2}\right)\left(\partial_{y} \psi\right)^{2}+2 x\left(1-y^{2}\right) \partial_{x} \psi \partial_{y} \psi\right]
\end{gathered}
$$

where $\partial_{a} \equiv \partial / \partial a$.

Equations (2)-(4) leave enough freedom in choosing the specific form of the metric potentials. The entering (1) constant $M$ is associated to the mass of the $\mathrm{BH}$.

A non-trivial generalization of (1), which includes the third metric potential $\Phi$, now dependent on $(y, \varphi)$ coordinates, therefore breaking the axisymmetric invariance of the original metric, is

$$
d s^{2}=-e^{2 \psi(x, y)} d t^{2}+M^{2} e^{-2 \psi(x, y)} \times\left[e^{2 \gamma(x, y)}\left(x^{2}-y^{2}\right)\left(\frac{d x^{2}}{x^{2}-1}+\frac{e^{\Phi(y, \varphi)} d y^{2}}{1-y^{2}}\right)+e^{\Phi(y, \varphi)}\left(x^{2}-1\right)\left(1-y^{2}\right) d \varphi^{2}\right]
$$

In this case, the presence of the third metric potential with the specific dependence on coordinates puts strong restrictions on the metric potentials $\psi(x, y)$ and $\gamma(x, y)$. In particular, the non-triviality of $\Phi(y, \varphi)$ results in the appearance of non-diagonal terms in the Ricci tensor that, in the absence of matter in the Einstein equations, sets the following constraints on $\gamma(x, y)$ :

$$
\partial_{x} \gamma=\frac{x\left(1-y^{2}\right)}{\left(x^{2}-y^{2}\right)\left(x^{2}-1\right)}, \quad \partial_{y} \gamma=\frac{y}{x^{2}-y^{2}},
$$

the further account of which leads to the additional restriction on $\psi(x, y)$ :

$$
\partial_{x} \psi \partial_{y} \psi=0
$$

A general solution to (6) comes as follows:

$$
\gamma(x, y)=\frac{1}{2} \ln \frac{x^{2}-1}{x^{2}-y^{2}}+C_{\gamma}
$$

then, solving for the corresponding equation for $\psi(x, y)$ constrained by (7), we get

$$
\psi=\frac{1}{2} \ln \frac{x-1}{x+1}+C_{\psi} .
$$

Note that (9) is a particular solution for $\psi$, which is convenient to choose in this form to establish in what follows the relation of (5) to a quasi-spherical Schwarzschild $\mathrm{BH}$ metric. With $\gamma$ and $\psi$ of (8), (9), the metric potential $\Phi(y, \varphi)$ is confined by the following differential equation:

$$
\partial_{y}\left(\left(y^{2}-1\right) \partial_{y} \Phi\right)-2\left(e^{\Phi}-1\right)+e^{2 C_{\gamma}} \frac{\partial_{\varphi}^{2} \Phi}{y^{2}-1}=0
$$


Fixing two integration constants $C_{\gamma}$ and $C_{\psi}$ to be zero and introducing new coordinates $(r, \theta)$, related to $(x, y)$ via

$$
x=\frac{r}{M}-1, \quad y=\cos \theta,
$$

we recover the metric of a neutral BH [63,64] (also see Ref. [65]) in the spherical coordinates

$$
d s^{2}=-f(r) d t^{2}+\frac{d r^{2}}{f(r)}+r^{2} e^{\chi(\theta, \varphi)}\left(d \theta^{2}+\sin ^{2} \theta d \varphi^{2}\right)
$$

with the standard red-shift factor $f(r)=1-2 M / r$ and the "smearing" function of the $\mathrm{BH}$ horizon $\chi(\theta, \varphi)$, which follows from $\Phi(y, \varphi)$ after the coordinate transformations (11). Equation (10) turns into the spherical Liouville equation

$$
\Delta_{\theta, \varphi} \chi(\theta, \varphi)+2\left(e^{\chi(\theta, \varphi)}-1\right)=0,
$$

with

$$
\Delta_{\theta, \varphi}=\frac{1}{\sin \theta} \frac{\partial}{\partial \theta} \sin \theta \frac{\partial}{\partial \theta}+\frac{1}{\sin ^{2} \theta} \frac{\partial^{2}}{\partial \varphi^{2}} .
$$

Recall that the Liouville equations are exactly-solvable and possess general analytic solutions in terms of unrestricted functions; see, e.g., Refs. [68,69], or a brief summary in Refs. $[61,64]$ and Appendix A in below. Therefore, we have enough freedom in choosing a function to fit the desired shape of a two-dimensional surface. For a BH, this surface is a quasi-spherical/distorted horizon; in the case of a rigid celestial body (as a neutron star), the two-dimensional surface is a 2-dimensional (2D) slice of the 3D surface, deformed by the tidal forces of another star/BH.

Therefore, we have established the generalization of the standard Weyl-Erez-Rosen distorted $\mathrm{BH}$ solution (1) with additional, breaking the axial symmetry, metric potential (cf. (5)). As a result of such modification, the solution becomes more rigid, and corresponds, after turning to the standard spherical coordinates, to the neutral $\mathrm{BH}$ solution with the Liouville mode [61-64]. That makes possible to consider the BH solution of Refs. [61-65] as a specific distorted $\mathrm{BH}$. Taking this point of view, we will not differentiate further on distorted and quasi-spherical BHs and will freely use both terms on equal footing.

To sum up this part of the work, the spacetime background we will consider throughout the paper is defined by the line element

$$
d s^{2}=-f(r) d t^{2}+\frac{d r^{2}}{f(r)}+r^{2} e^{\chi(\theta, \varphi)}\left(d \theta^{2}+\sin ^{2} \theta d \varphi^{2}\right),
$$

with

$$
f(r)=\frac{\Delta}{r^{2}}, \quad \Delta=r^{2}-2 M r+\kappa^{2} r^{4}
$$

A non-trivial value of $\kappa^{2}=-\Lambda / 3$, where $\kappa$ is the inverse characteristic length of AdS spacetime and $\Lambda$ is its cosmological constant, corresponds to the AdS-Schwarzschild BH; $\kappa=0$ is that of the flat spacetime. To solve the vacuum Einstein equations, the metric potential $\chi(\theta, \varphi)$ has to be confined to the spherical Liouville Equation (13) with the angular Laplacian (14).

\section{Basic Equations and Separation of Variables}

Technically, we would like to extend the known Regge-Wheeler-Zerilli [70,71] equations for small d-wave perturbations over the background (15) and to solve them. First step on this way is to separate the variables in the corresponding relativistic spin equation. Our previous experience with s-wave perturbations over such a background [64] indicates 
the possibility to separate variables in the massless Klein-Gordon equation, the explicit form of which in the background (15) is as follows:

$$
-\frac{1}{f} \partial_{t}^{2} \Phi+\frac{1}{r^{2}} \partial_{r}\left(r^{2} f \partial_{r} \Phi\right)+\frac{e^{-\chi(\theta, \varphi)}}{r^{2}} \triangle_{\theta, \varphi} \Phi=0,
$$

by use of the separation ansatz

$$
\Phi(t, r, \theta, \varphi)=e^{-i \omega t} Q_{0}(r) \Theta(\theta, \varphi)
$$

But it is well-known that the (im)possibility to separate the variables strongly depends on special properties of the spacetime upon the consideration, specifically on its type within the Petrov spacetime classification scheme [72,73].

To determine the Petrov type of the metric in hand, we follow the Newman-Penrose (NP) formalism [74] and introduce the null tetrad

$$
e_{(1)}^{\mu}=l^{\mu}, e_{(2)}^{\mu}=n^{\mu}, e_{(3)}^{\mu}=m^{\mu}, e_{(4)}^{\mu}=\bar{m}^{\mu},
$$

which is standardly related to the metric via $g_{\mu v}=e_{\mu}^{(a)} \eta_{(a)(b)} e_{\nu}^{(b)}$ with

$$
\eta_{(a)(b)}=\left(\begin{array}{cccc}
0 & -1 & 0 & 0 \\
-1 & 0 & 0 & 0 \\
0 & 0 & 0 & 1 \\
0 & 0 & 1 & 0
\end{array}\right)
$$

The curved space indices $\mu, v$ run over $0,1,2,3$; " 0 " corresponds to time direction. The latin indices " $(a)$ " are that of flat tangent space and run over 1,2,3, 4 .

Explicitly, for the metric (15), we get

$$
l_{\mu}=\delta_{\mu 0}-\frac{\delta_{\mu r}}{f(r)}, \quad n_{\mu}=\frac{f(r)}{2} \delta_{\mu 0}+\frac{\delta_{\mu r}}{2}, m_{\mu}=\frac{r}{\sqrt{2}} e^{\frac{\chi(\theta, \varphi)}{2}}\left(\delta_{\mu \theta}+i \sin \theta \delta_{\mu \varphi}\right)
$$

where $\delta_{\mu \nu}$ is the Kronecker delta.

By use of the NP tetrad (21), one may compute various coefficients of the spinconnection $\gamma_{(c)(a)(b)}=e_{(c)}^{v} e_{(a) v ; \mu} e_{(b)}^{\mu}$, where, as usual, the semicolon corresponds to the covariant derivative over the metric $g_{\mu \nu}$, and observe vanishing the coefficients (in the notation of Ref. [74]) $\kappa, \sigma, v, \lambda$. According to the Goldberg-Sachs theorem, trivialization of $\kappa, \sigma, v, \lambda$ corresponds to the Petrov type D metrics [72-74].

Since the pioneering papers by Teukolsky $[75,76]$, it was realized that, in the Petrov type D spacetimes, equations for gravitational perturbations decouple for quantities

$$
\psi_{0}=-C_{\alpha \beta \gamma \delta} l^{\alpha} m^{\beta} l^{\gamma} m^{\delta}, \quad \psi_{4}=-C_{\alpha \beta \gamma \delta} n^{\alpha} \bar{m}^{\beta} n^{\gamma} \bar{m}^{\delta},
$$

forming with the Weyl tensor $C_{\alpha \beta \gamma \delta}$ and the NP tetrad (21). As we will see, in short, these quantities correspond to the odd (axial) gravitational perturbation of Ref. [70] and even (polar) d-wave perturbation of Ref. [71].

Indeed, by use of the ansatz (we use indices " +2 " and " -2 " for odd and even d-wave perturbations, respectively)

$$
\left(\begin{array}{c}
\psi_{0} \\
r^{4} \psi_{4}
\end{array}\right)=e^{-i \omega t}\left(\begin{array}{c}
\Psi_{+2}(r) \Theta_{+2}(\theta, \varphi) \\
\Psi_{-2}(r) \Theta_{-2}(\theta, \varphi)
\end{array}\right)
$$

one may separate the temporal, radial and angular parts of the gravitational perturbations over the basic metric (15). Upon the separation of the angular part of the d-wave pertur- 
bations, we arrive at the following master equation for the fundamental angular variable $\Theta(\theta, \varphi)$ :

$$
\Delta_{\theta, \varphi} \Theta(\theta, \varphi)+C e^{\chi(\theta, \varphi)} \Theta(\theta, \varphi)=0,
$$

in which, for the sake of convenience in further comparing to the spherically symmetric example, we set the separation constant to $C=v(v+1)$. Apparently, the spherical symmetry of the background spacetime is recovered for the trivial metric potential $\chi(\theta, \varphi)=0$ $(\chi(\theta, \varphi)=$ const also gets, re-scaling the radial coordinate, the spherical symmetry back). However, in general, the spherical symmetry is lost, that means $v$ does not fall into the set of integers. The fundamental angular variable $\Theta(\theta, \varphi)$, together with angular differential operators

$$
L_{n}=\partial_{\theta}-\frac{i}{\sin \theta} \partial_{\varphi}+n\left(\cot \theta+\frac{1}{2}\left(\partial_{\theta} \chi-\frac{i}{\sin \theta} \partial_{\varphi} \chi\right)\right),
$$

form $\Theta_{ \pm 2}(\theta, \varphi)$ of $(23)$ :

$$
\begin{aligned}
& \Theta_{+2}(\theta, \varphi)=e^{-\frac{1}{2} \chi(\theta, \varphi)} L_{-1}^{\dagger} e^{-\frac{1}{2} \chi(\theta, \varphi)} L_{0}^{\dagger} \Theta(\theta, \varphi), \\
& \Theta_{-2}(\theta, \varphi)=e^{-\frac{1}{2} \chi(\theta, \varphi)} L_{-1} e^{-\frac{1}{2} \chi(\theta, \varphi)} L_{0} \Theta(\theta, \varphi) .
\end{aligned}
$$

For the radial part of the perturbation equations of $\psi_{0}$ and $\psi_{4}$, the NP formalism is equivalent (after transition to new radial functions $Q_{ \pm 2}(r)$; see Refs. [77,78] for details) to the Regge-Wheeler-Zerilli equations

$$
\left[\frac{\partial^{2}}{\partial r_{*}^{2}}+\omega^{2}-V_{s}(r)\right] Q_{s}=0, \quad r_{*} \in(-\infty,+\infty), \quad s= \pm 2
$$

Here, $r_{*}$ is the so-called "tortoise" coordinate $d r / d r_{*}=f(r) ; V_{ \pm 2}$ are the effective potentials, entering either the Regge-Wheeler [70]

$$
V_{+2}(r)=-\frac{3 f(r) \partial_{r} f(r)}{r}+v(v+1) \frac{f(r)}{r^{2}}+6 \kappa^{2} f(r),
$$

or the Zerilli [71]

$$
V_{-2}(r)=\frac{2 f(r)}{r^{3}} \frac{9 M^{3}+3 c^{2} M r^{2}+c^{2}(1+c) r^{3}+9 M^{2}\left(c r+3 \kappa^{2} r^{3}\right)}{(3 M+c r)^{2}}, \quad c=\frac{v(v+1)}{2}-1
$$

equations. Altogether, the Regge-Wheeler-Zerilli Equation (27) look like a stationary Schrödinger equation for the wave functions $Q_{ \pm 2}$ and the effective potentials (28) and (29). Note that, due to the lack of the spherical symmetry, we do not have the total angular momentum (and its projection, as well) conservation, though we can expect a quantization of the generalized angular momentum quantum numbers $v$.

Let us also emphasize that the scalar and vector perturbations over the basic metric (15) are described by a Schrödinger-like Equation (27), as well, with the effective potential (33), in which one has to choose $s=0$ and $s=1$ for the scalar and vector modes, respectively. The common separation ansatz for the scalar, vector, and tensor perturbations looks as follows (cf. (23)):

$$
\left(\begin{array}{c}
r^{-1} \Phi \\
r \tilde{\phi} \\
\psi_{0} \\
r^{4} \psi_{4}
\end{array}\right)=e^{-i \omega t}\left(\begin{array}{c}
Q_{0}(r) \Theta(\theta, \varphi) \\
Q_{1}(r) \Theta_{1}(\theta, \varphi) \\
\Psi_{+2} \Theta_{+2}(\theta, \varphi) \\
\Psi_{-2} \Theta_{-2}(\theta, \varphi)
\end{array}\right)
$$

The $\Theta(\theta, \varphi)$ function of (30) obeys the master Equation (24); $\Theta_{ \pm 2}(\theta, \varphi)$ are those of (26). $\Theta_{1}(\theta, \varphi)$ is also related to the fundamental angular variable $\Theta(\theta, \varphi)$; in this case we have

$$
\Theta_{1}=e^{-\frac{1}{2} \chi(\theta, \varphi)} L_{1}^{\dagger} \Theta(\theta, \varphi),
$$


with the angular differential operator $L_{1}$ of (25). Therefore, the "axial" perturbations over the quasi-spherical $\mathrm{BH}$ background are described by equations

$$
\left[\frac{\partial^{2}}{\partial r_{*}^{2}}+\omega^{2}-V_{s}(r)\right] Q_{s}=0, \quad r_{*} \in(-\infty,+\infty), s=0,1,2,
$$

with the effective potentials [24-26,70,79-81]

$$
V_{s}(r)=\frac{\left(1-s^{2}\right) f \partial_{r} f}{r}+v(v+1) \frac{f}{r^{2}}+3 s(s-1) \kappa^{2} f, \quad s=0,1,2 .
$$

The polar d-wave perturbation is still described by the Zerilli Equation (27) with $s=-2$ and with the effective potential (29). The shape of the effective potentials for flat and AdS spacetimes in the spherically symmetric case are depicted in Figures 1 and 2.

Two comments on Figures 1 and 2 are in order. First, the shape of the effective potentials in Minkowski space-time (both panels of Figure 1) keeps its form up to spatial (radial in the case) infinity. It is not true for AdS spacetime, where the shape of the potentials (in both panels of Figure 2) will change to $\sim r^{2}$ profile at $r \gg 1$. It, in particular, means that there are bound states in AdS spacetime that result in the discrete part of the spectrum of admissible perturbations [38,64,81-83]. And second, the effective potentials of the ReggeWheeler-Zerilli Equation (28) and (29) are almost the same as in Minkowski, as well as in AdS spacetimes (cf. right panels in Figures 1 and 2). This is the indication of isospectrality of the Hamiltonians, entering Equation (27) [81,84-86].
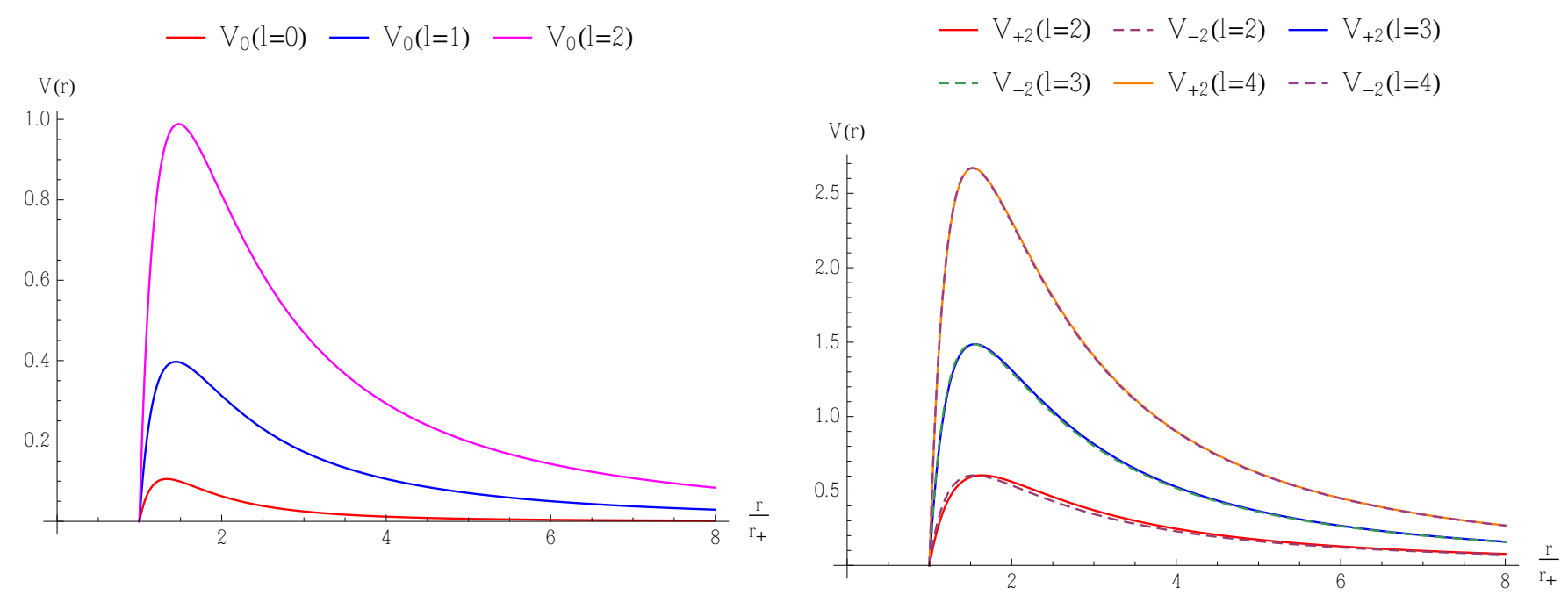

Figure 1. (Left panel) The shape of the effective potential of different modes $(l=0,1,2)$ of the scalar perturbation over the spherically-symmetric Schwarzschild background. (Right panel) Comparison of the effective potentials $V_{+2}(r)$ (solid lines) and $V_{-2}(r)$ (dashed lines) of the d-wave perturbations $(l=2,3,4)$ over the spherically-symmetric Schwarzschild background. The (almost) coincidence of $V_{ \pm 2}$ reflects the isospectrality of the corresponding Hamiltonians of (27). The value of the horizon radial location is chosen to be $r_{+}=1$. 

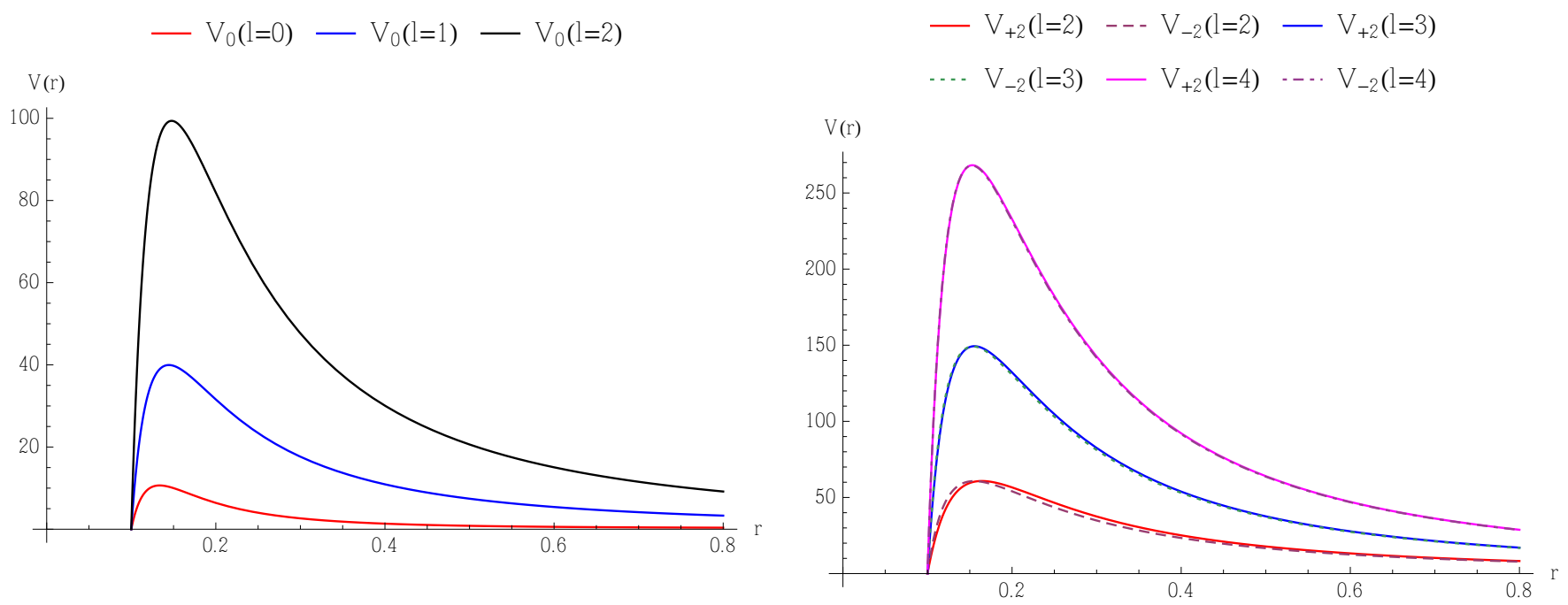

Figure 2. (Left panel) The shape of the effective potential of different modes $(l=0,1,2)$ of the scalar perturbation over the spherically-symmetric Anti-de Sitter (AdS)-Schwarzschild background near the black hole (BH) horizon. (Right panel) Comparison of the effective potentials $V_{+2}(r)$ (solid lines) and $V_{-2}(r)$ (dashed lines) of the d-wave perturbations $(l=2,3,4)$ over the spherically-symmetric AdS-Schwarzschild background near the BH horizon. The value of the horizon radial location is $r_{+}=0.1$. The AdS cosmological constant is chosen to be $\kappa^{2}=-\Lambda / 3=0.1$.

4. Generalized Angular Momentum Numbers: Generalities and Axial Symmetry Case

Let us turn back to the master equation for the fundamental angular variable (24). It can be solved with series expansion of $\Theta(\theta, \varphi)$ over the spherical harmonics $Y_{l m}$ :

$$
\Theta(\theta, \varphi)=\sum_{l, m}^{\infty} c_{l m} Y_{l m}(\theta, \varphi) ; \quad l \in \mathbb{Z}, m=-l, \ldots, l .
$$

For a general function of angles, the expansion (34) contains infinite number of terms. Therefore, once one plugs (34) in the master Equation (24), the solution to the master equation turns into a generalized eigenvalue problem:

$$
\sum_{j, m}^{\infty} A_{k m^{\prime}, j m} c_{j m}=C \sum_{j, m}^{\infty} B_{k m^{\prime}, j m} c_{j m}, \quad C=v(v+1),
$$

with infinite dimensional matrices

$$
A_{k m^{\prime}, j m}=j(j+1) \delta_{k j} \delta_{m^{\prime} m}, \quad B_{k m^{\prime}, j m}=\int d \Omega e^{\chi(\theta, \varphi)} Y_{k m^{\prime}}^{*}(\theta, \varphi) Y_{j m}(\theta, \varphi) .
$$

As usual, the measure of integration over the angle variables is determined by $d \Omega=$ $\sin \theta d \theta d \varphi$.

One of the ways to resolve the generalized eigenvalue problem we are dealing with is to use numerical computations. However, even in this case, we have to establish an upper bound for $j$ in (35) to reduce the task to the generalized eigenvalue problem of $n \times n$ matrices $\mathbf{A}$ and $\mathbf{B}$

$$
\mathbf{A} \cdot \mathbf{c}=C \mathbf{B} \cdot \mathbf{c},
$$

where

$$
n=\sum_{j=0}^{j_{\max }}(2 j+1)=\left(1+j_{\max }\right)^{2} .
$$

The upper bound value $j_{\text {max }}$ is chosen in such a way that the eigenvalues and the corresponding eigenvectors do not visibly change upon $j_{\max }$ increasing. 
Another simplification can be reached with restoring a part of the spherical symmetry, that is the axial symmetry. Viz., the metric potential $\chi$, generally dependent on two spherical angles, becomes a function of just one polar angle $\theta$.

In this case, Equation (13) simplifies to

$$
\frac{1}{\sin \theta} \frac{d}{d \theta}\left(\sin \theta \frac{d \chi(\theta)}{d \theta}\right)+2\left(e^{\chi(\theta)}-1\right)=0
$$

so one can establish that (see Appendix A)

$$
e^{\chi(x)}=\frac{(2 a b)^{2}\left(\frac{1-x}{1+x}\right)^{a}}{\left(1-x^{2}\right)\left(b^{2}+\left(\frac{1-x}{1+x}\right)^{a}\right)^{2}}, \quad x=\cos \theta,
$$

where the constants $a=1+\alpha$ and $b=1+\beta$ are restricted to be real and positive. (The case of trivial deformation parameters $\alpha$ and $\beta$ corresponds to the spherical symmetry of the background metric.) Additional restriction on the deformation parameters is required by finiteness of the metric potential $e^{\chi(\theta)}$ in the endpoints of the $\theta$ fundamental domain $\theta \in[0, \pi]$. According to this requirement, $\alpha$ has to be non-negative.

Axial symmetry makes possible to further separate the angular variables

$$
\Theta \rightarrow \Theta_{m}(\theta, \varphi)=e^{i m \varphi} S_{m}(\theta), \Theta_{m}(\theta, \varphi)=\Theta_{m}(\theta, \varphi+2 \pi) \rightarrow m \in \mathbb{Z},
$$

so that the master Equation (24) is reduced to the enlargement of general Legendre equation:

$$
\frac{d}{d x}\left[\left(1-x^{2}\right) \frac{d S_{m}(x)}{d x}\right]+\left[v(v+1) e^{\chi(x)}-\frac{m^{2}}{1-x^{2}}\right] S_{m}(x)=0, \quad x=\cos \theta .
$$

Consequently, the matrices (36) are reduced to

$$
A_{i j}^{m}=j(j+1) \frac{2(j+m) !}{(2 j+1)(j-m) !} \delta_{i j}, \quad B_{i j}^{m}=\int_{-1}^{1} d x e^{\chi(x)} P_{i m}(x) P_{j m}(x),
$$

and the generalized eigenvalue problem (35) turns into

$$
\sum_{j=|m|}^{\infty} A_{i j}^{m} c_{j}=C \sum_{j=|m|}^{\infty} B_{i j}^{m} c_{j}
$$

Solving for Equation (44), we arrive at the following conclusions: the eigenvalues $v^{\prime} \mathrm{s}$ are labeled with two indices- $l \geq 0$ and $m=\{-l, \ldots, l\}$-with the trivialization condition $v_{l m} \rightarrow l \in \mathbb{Z}$ once $\chi \rightarrow 0$; numerics also give $v_{l 0}=l$ and $v_{l,-m}=v_{l m}$. Computations of the separation constant $C_{l m}=v_{l m}\left(v_{l m}+1\right)$ for different values of the admissible deformation degrees $\alpha$ and $\beta$ show the independence of the results from the value of $\beta$. See Table 1 as an example. Therefore, $v_{l m}$ do not depend on $\beta$ and are solely functions of the parameter $\alpha$. 
Table 1. Values of $C_{l m}=v_{l m}\left(v_{l m}+1\right)$ with $l=1, m=1$ for different deformation degrees $\alpha$ and $\beta$ obtained by solving for Equation (44) with different cut-off values of $j_{\max }$.

\begin{tabular}{cccc}
\hline$\alpha$ & $\beta$ & $j_{\max }=42$ & $j_{\max }=\mathbf{7 2}$ \\
\hline$\alpha=0.001$ & $\beta=0.001$ & 1.99873 & 1.99700 \\
$\alpha=0.001$ & $\beta=0.01$ & 1.99873 & 1.99700 \\
$\alpha=0.001$ & $\beta=0.1$ & 1.99873 & 1.99700 \\
$\alpha=0.001$ & $\beta=1.0$ & 1.99873 & 1.99700 \\
\hline$\alpha=0.01$ & $\beta=0.001$ & 1.97164 & 1.97039 \\
$\alpha=0.01$ & $\beta=0.01$ & 1.97164 & 1.97039 \\
$\alpha=0.01$ & $\beta=0.1$ & 1.97164 & 1.97039 \\
$\alpha=0.01$ & $\beta=1.0$ & 1.97164 & 1.97039 \\
\hline$\alpha=0.1$ & $\beta=0.001$ & 1.73554 & 1.73553 \\
$\alpha=0.1$ & $\beta=0.01$ & 1.73554 & 1.73553 \\
$\alpha=0.1$ & $\beta=0.1$ & 1.73554 & 1.73553 \\
$\alpha=0.1$ & $\beta=1$ & 1.73554 & 1.73553 \\
\hline$\alpha=1.0$ & $\beta=0.001$ & 0.74999 & 0.74999 \\
$\alpha=1.0$ & $\beta=0.1$ & 0.74999 & 0.74999 \\
$\alpha=1.0$ & $\beta=1$ & 0.74999 & 0.74999 \\
$\alpha=1.0$ & & 0.74999 & 0.74999 \\
\hline
\end{tabular}

Next, fixing $b=1$ and $a=1+\alpha$, we observe from numerics that first eigenvalues with $l, m=1,2$ as functions of a positive parameter $\alpha$ obey the inequality $v_{l m} \leq l$ (see Figure 3). This trend gets preserved for other combinations of $(l, m)$ in $v_{l m}$, as well. (Cf. Table 2.)

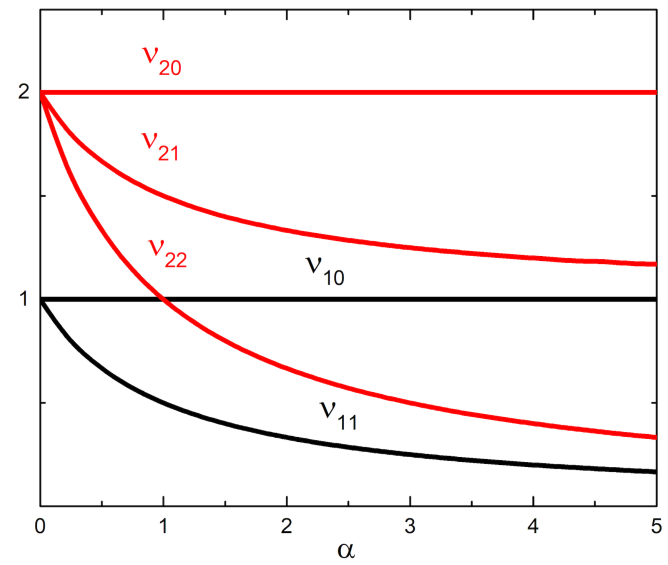

Figure 3. Eigenvalues $v_{l m}$ for $l=1,2$ and $m \leq l$.

Finally, comparing different values of $v_{l m}$ for different values of the deformation degree $\alpha$, we observe that (see Table 2)

$$
v_{l m}=v_{l-1, m}+1
$$

Therefore, we conclude that the angular momentum is quantized, but not in integers. 
Table 2. Values of $v_{l m}(l=0, \ldots, 5, m=0, \ldots, 4)$ for different values of the deformation parameter $\alpha$.

\begin{tabular}{ccccccc}
\hline & $\boldsymbol{\alpha}=\mathbf{0 . 0 0 1}$ & $\boldsymbol{\alpha}=\mathbf{0 . 0 1}$ & $\boldsymbol{\alpha}=\mathbf{0 . 1}$ & $\boldsymbol{\alpha}=\mathbf{1}$ & $\boldsymbol{\alpha}=\mathbf{2}$ & $\boldsymbol{\alpha}=\mathbf{3}$ \\
\hline$v_{00}$ & 0 & 0 & 0 & 0 & 0 & 0 \\
$v_{10}$ & 1 & 1 & 1 & 1 & 1 & 1 \\
$v_{20}$ & 2 & 2 & 2 & 2 & 2 & 2 \\
$v_{30}$ & 3 & 3 & 3 & 3 & 3 & 3 \\
$v_{40}$ & 4 & 4 & 4 & 4 & 4 & 4 \\
$v_{50}$ & 5 & 5 & 5 & 5 & 5 & 5 \\
\hline$v_{11}$ & 0.999 & 0.990 & 0.909 & 0.5 & 0.333 & 0.25 \\
$v_{21}$ & 1.999 & 1.990 & 1.909 & 1.5 & 1.333 & 1.25 \\
$v_{31}$ & 2.999 & 2.990 & 2.909 & 2.5 & 2.333 & 2.25 \\
$v_{41}$ & 3.999 & 3.990 & 3.909 & 3.5 & 3.333 & 3.25 \\
$v_{51}$ & 4.999 & 4.990 & 4.909 & 4.5 & 4.333 & 4.25 \\
\hline$v_{22}$ & 1.9980 & 1.9802 & 1.8181 & 1 & 0.666 & 0.5 \\
$v_{32}$ & 2.9980 & 2.9802 & 2.818 & 2 & 1.666 & 1.5 \\
$v_{42}$ & 3.9980 & 3.9802 & 3.818 & 3 & 2.666 & 2.5 \\
$v_{52}$ & 4.9980 & 4.9802 & 4.818 & 4 & 3.666 & 3.5 \\
\hline$v_{33}$ & 2.9970 & 2.9702 & 2.727 & 1.5 & 1.0 & 0.75 \\
$v_{43}$ & 3.9970 & 3.9702 & 3.727 & 2.5 & 2.0 & 1.75 \\
$v_{53}$ & 4.9970 & 4.9702 & 4.727 & 3.5 & 3.0 & 2.75 \\
\hline$v_{44}$ & 3.9960 & 3.9604 & 3.6363 & 2 & 1.333 & 1.0 \\
$v_{54}$ & 4.9960 & 4.9604 & 4.6363 & 3 & 2.333 & 2.0 \\
\hline
\end{tabular}

We end up this section with an analysis of Table 2, which results in the following analytical expression for $v_{l m}(\alpha)$ :

$$
v_{l m}(\alpha)=l-\frac{\alpha}{1+\alpha} m
$$

The obtained expression is in the fine agreement with the previously numerically computed functional dependences of $v_{l m}$ on $\alpha$ (Figure 3). Let us also notice the coincidence in $v_{l m}=v_{l+k, m+s}$ values for integers $(k, s)$, determined by $k(1+\alpha)=\alpha s$ and restricted by $l+k \geq m+s$. For instance, $v_{00}=v_{33}, v_{20}=v_{43}=v_{66}, v_{21}=v_{44}, v_{31}=v_{54}=v_{77}$, and so on.

\section{The Grey-Body Factor: Schwarzschild vs. Distorted Schwarzschild}

Now, let us turn to the radial part of the d-wave perturbations described by Equation (27). Solving for these equations analytically is hampered due to a complicated form of the effective potentials. Typically, one has to find solutions either numerically (as it was done in Ref. [43]), or to follow the procedure of finding solutions in different coordinate domains — the near, mid and far zones (see, e.g., Ref. [38], for a review) —with subsequent constructing the united solution in different approximations (as, for instance, in Refs. [23,25-29]). In the context of the scattering problem, solutions to Equation (27) are used in computing different cross-sections, one of important ingredients of which is the so-called grey-body factor (GBF). To compute this characteristic, one has to solve a Schrödinger-like Equation (27) with the specified boundary conditions (see, for example, Ref. [38]), which especially determine the transmission coefficient of an ingoing wave through the barrier of effective potentials (28) or (29). Then, the grey-body factor is $\gamma(\omega)=|T(\omega)|^{2}$, where $T(\omega)$ stands for the transmission coefficient. The complete transmission means the complete absorption of incoming waves by a $\mathrm{BH}$.

To figure out hallmarks of scattering/absorption in the background of quasispherical/distorted BHs, we will compare the spherically symmetric case (here, we follow the approach of Ref. [43]) with that of deformed but axially symmetric. Looking at Figure 4 with the results of numerics for the spherically symmetric (Schwarzschild) background (which reproduce in part data in Figures 2 and 8 of Ref. [43]), one may notice that: 
- the scalar s-wave (left panel) has the complete transmission at the lowest admissible value of frequency $\omega$;

- $\quad$ increasing $l$ in the scalar mode perturbations ( $\mathrm{p}$ - and d-modes on the left panel) requires higher values of $\omega$ to reach the complete transmission;

- basic axial gravitational perturbations ( $d$-waves) (right panel) reach the complete transmission at lower, w.r.t. $l=2$ mode of axial electro-magnetic (EM) and scalar perturbations, frequency.
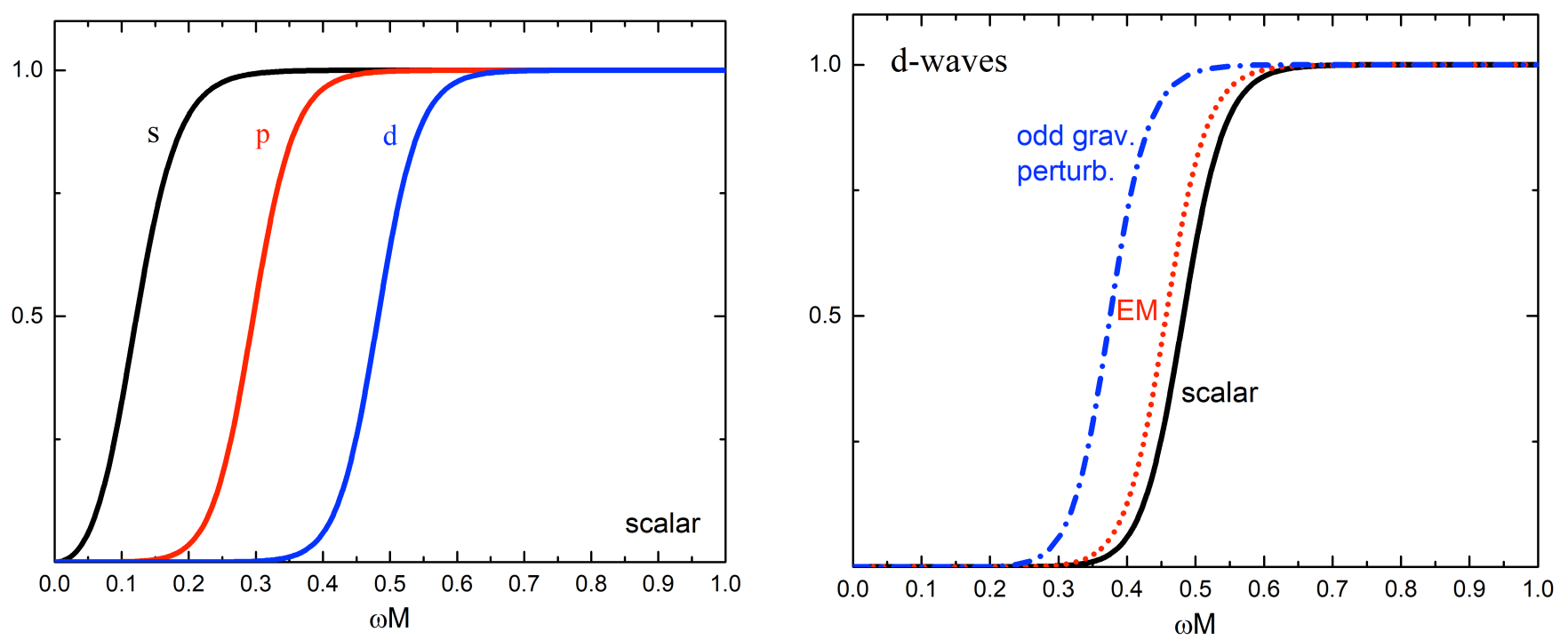

Figure 4. (Left panel) The grey-body factors $\gamma(\omega)$ of different modes $(l=0,1,2)$ of the scalar perturbation over the Schwarzschild background. (Right panel) Comparison of $\gamma(\omega)$ of the basic axial d-wave perturbation $(l=2)$ to the corresponding mode (with $l=2$ ) grey-body factors (GBFs) of the scalar and axial electro-magnetic (EM) perturbations over the Schwarzschild background.

For the distorted BH background (15) with the metric potential (40), the deformation parameters of which are chosen to be $b=1$ and $a=1+\alpha=1+0.2$, putting the data for the same type of waves on plots, we encounter important differences in compare to the previously considered cases (see Figure 5 ). We observe that:

- $\quad$ for each value of $l$ (recall, $l \in \mathbb{Z}$ is a non-negative degree of the corresponding spherical harmonics in the series expansion (34)), there are $l+1$ different values of the grey-body factor $\gamma(\omega)$;

- $\gamma(\omega)$ gets increased with increasing the deformation degree $\alpha$;

- for a fixed $l$, the GBFs with the maximal projection value $m=l$ reach the complete transmission at the lowest values of $\omega$. 
$\gamma(\omega)$

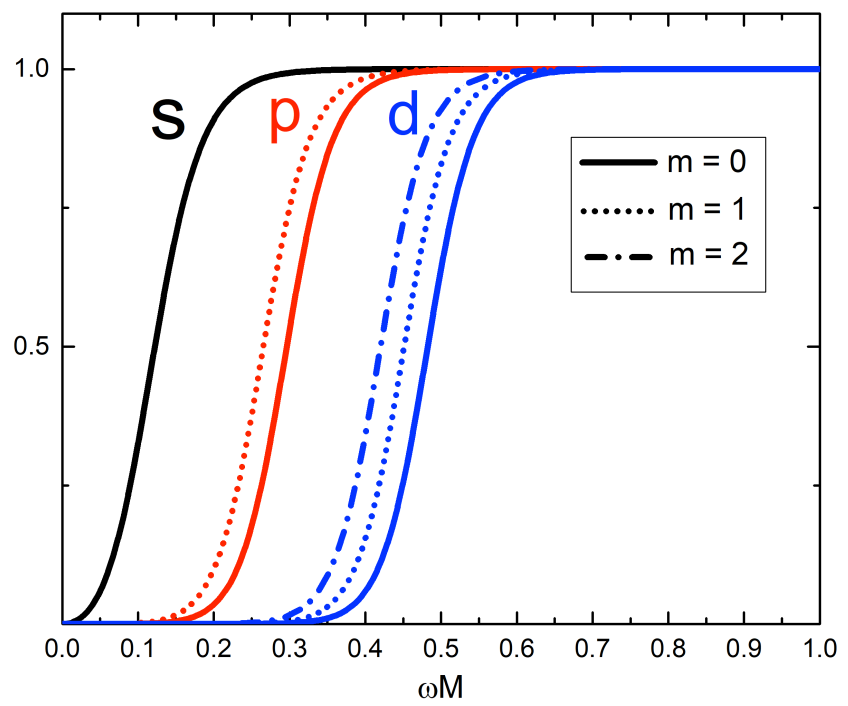

$\gamma(\omega)$

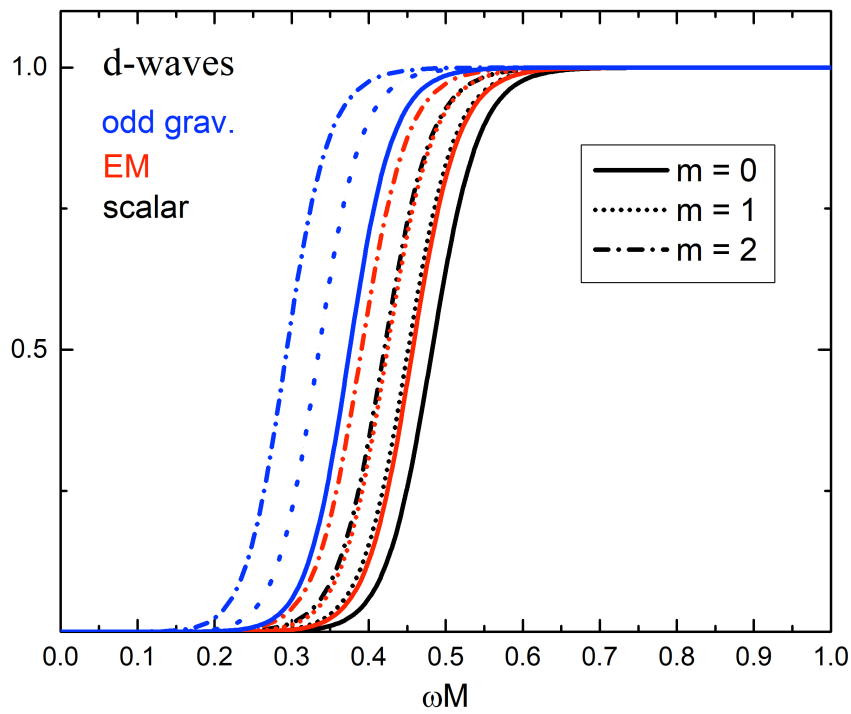

Figure 5. (Left panel) Splitting the grey-body factors $\gamma(\omega)$ for different modes $(l=0,1,2)$ of the scalar perturbation in the distorted BH background. (Right panel) Comparison of the GBFs of the basic axial d-wave perturbation $(l=2)$ to that of the scalar and axial electro-magnetic (EM) perturbations over the distorted BH background. The deformation parameter $\alpha$ is equal to 0.2 .

\section{Quasinormal Modes of a Quasi-Spherical Axisymmetric Black Hole}

As we have noted above, the scattering problem for an axially-symmetric neutral $\mathrm{BH}$ is completely determined by a single parameter - the deformation degree $\alpha$ - which is required to be non-negative without any additional limitations. However, new restrictions on $\alpha$ could appear from the demand on stability of the $\mathrm{BH}$ spacetime background against small perturbations. That is fully determined by the quasinormal modes (QNMs).

Recall that the quasinormal modes (see, e.g., Refs. [77-81,83,86-95]) correspond to solutions to Equations (27) and (32), which satisfy the specific boundary conditions (cf. $[77,79,80])$ :

ingoing waves at the horizon

$$
Q_{S}(x) \simeq e^{-i \omega r_{*}}, \quad r_{*} \rightarrow-\infty\left(r \rightarrow r_{+}\right),
$$

and outgoing waves at the spatial infinity

$$
\begin{aligned}
& Q_{S}(x) \simeq e^{i \omega r_{*}}, \quad r_{*}, r \rightarrow+\infty \quad \text { flat space } \\
& Q_{S}(x) \rightarrow 0, \quad r_{*}, r \rightarrow+\infty \quad \text { AdS space }
\end{aligned}
$$

For fixed values of spin $s$ and (projection of) angular momentum $(l, m)$, there are infinite number of QNMs, which are labeled by the overtone number $n$; the least damped (fundamental) QNMs correspond to $n=0$.

Generally, the QNMs are complex and their imaginary part can be positive or negative. It is easy to verify $[79,80]$ that positivity in the imaginary part of any QNM results in the instability of the background geometry. Indeed, following Refs. [79,80], we will find solutions to the Schrödinger-like equation

$$
\left[\frac{\partial^{2}}{\partial r_{*}^{2}}+\omega^{2}-V_{s}(r)\right] Q_{s}=0, \quad r_{*} \in(-\infty,+\infty),
$$

with the "wave-function"

$$
Q_{s}(r)=e^{-i \omega r_{*}} \phi_{s}(r),
$$


and figure out restrictions on the admissible complex frequencies. To maintain the boundary conditions (47), (48), the "amplitude" $\phi_{s}(r)$ should satisfy

$$
\phi_{s}(r) \simeq \text { const }, \quad r \rightarrow r_{+}
$$

and

$$
\begin{aligned}
& \phi_{s}(r) \rightarrow e^{2 i \omega r_{*}}, \quad r_{*}, r \rightarrow+\infty \quad \text { flat space, } \\
& \phi_{s}(r) \rightarrow 0, \quad r_{*}, r \rightarrow+\infty \quad \text { AdS space }
\end{aligned}
$$

Plugging the ansatz (50) into Equation (49), for inherently complex valued $\phi_{s}(r)$, we get

$$
f \frac{d^{2} \phi_{s}}{d r^{2}}+\left(\frac{d f}{d r}-2 i \omega\right) \frac{d \phi_{s}}{d r}-\frac{V_{s}}{f} \phi_{s}=0, \quad f=1-\frac{2 M}{r}+\kappa^{2} r^{2} .
$$

Now, one multiplies both sides of (53) by $\phi_{s}^{*}(r)$ and integrates over $\left[r_{+}, \infty\right)$ :

$$
\int_{r_{+}}^{\infty} d r\left(\phi_{s}^{*} \frac{d\left(f \partial_{r} \phi_{s}\right)}{d r}-2 i \omega \phi_{s}^{*} \frac{d \phi_{s}}{d r}-\frac{V_{s}}{f}\left|\phi_{s}\right|^{2}\right)=0 .
$$

Further integration by parts on account of the boundary conditions (51), (52) and the asymptotic behaviour of the red-shift factor $f(r)$ at the integration end points results in the following expression for the first term of the integrand in (54):

$$
\int_{r_{+}}^{\infty} d r \frac{d\left(\phi_{s}^{*} f \partial_{r} \phi_{s}\right)}{d r}=\left.\phi_{s}^{*} f \frac{d \phi_{s}}{d r}\right|_{r=r_{+}} ^{r=\infty}=\left\{\begin{array}{lll}
2 i \omega & \text { flat } \\
0 & \text { AdS }
\end{array}\right.
$$

Hence,

$$
\int_{r_{+}}^{\infty} d r\left(f\left|\frac{d \phi_{s}}{d r}\right|^{2}+2 i \omega \phi_{s}^{*} \frac{d \phi_{s}}{d r}+\frac{V_{s}}{f}\left|\phi_{s}\right|^{2}\right)=\left\{\begin{array}{ll}
2 i \omega & \text { flat } \\
0 & \text { AdS }
\end{array},\right.
$$

and, for the imaginary part of (56), we obtain

$$
\int_{r_{+}}^{\infty} d r\left(\omega \phi_{s}^{*} \frac{d \phi_{s}}{d r}+\omega^{*} \phi_{s} \frac{d \phi_{s}^{*}}{d r}\right)=\left\{\begin{array}{ll}
\omega+\omega^{*} & \text { flat } \\
0 & \text { AdS }
\end{array} .\right.
$$

Using the integration by parts for the left-hand-side (l.h.s.) of (57) once again results in

$$
\int_{r_{+}}^{\infty} d r\left(\omega \phi_{s}^{*} \frac{d \phi_{s}}{d r}+\omega^{*} \phi_{s} \frac{d \phi_{s}^{*}}{d r}\right)=\left(\omega-\omega^{*}\right) \int_{r_{+}}^{\infty} \phi_{s}^{*} \frac{d \phi_{s}}{d r} d r+\left.\omega^{*}\left|\phi_{s}(r)\right|^{2}\right|_{r=r_{+}} ^{r=\infty},
$$

so that

$$
\int_{r_{+}}^{\infty} \phi_{s}^{*} \frac{d \phi_{s}}{d r} d r=\left\{\begin{array}{ll}
\frac{\omega+\omega^{*}\left|\phi_{s}\left(r_{+}\right)\right|^{2}}{\omega-\omega^{*}} & \text { flat } \\
\frac{\omega^{*}\left|\phi_{s}\left(r_{+}\right)\right|^{2}}{\omega-\omega^{*}} & \text { AdS }
\end{array} .\right.
$$

Lastly, substituting (59) into the 1.h.s. of (56) leads to $[79,80]$

$$
\int_{r_{+}}^{\infty} d r\left(f\left|\frac{d \phi_{s}}{d r}\right|^{2}+\frac{V_{s}}{f}\left|\phi_{s}\right|^{2}\right)=\left\{\begin{array}{ll}
-\frac{|\omega|^{2}\left|\phi_{s}\left(r_{+}\right)\right|^{2}+(\operatorname{Re} \omega)^{2}+(\operatorname{Im} \omega)^{2}}{\operatorname{Im} \omega} & \text { flat } \\
-\frac{|\omega|^{2}\left|\phi_{s}\left(r_{+}\right)\right|^{2}}{\operatorname{Im} \omega} & \text { AdS }
\end{array} .\right.
$$

Therefore, for a non-negative in the domain $\left[r_{+}, \infty\right)$ potential $V_{s}(r)$ (cf. Figures 1 and 2 with effective potentials in spherically-symmetric spacetimes), the 1.h.s. of (60) is nonnegative. The same is required for the right-hand-side of this expression that means negativity of the denominator: $\operatorname{Im} \omega<0$. Once the imaginary part of frequencies becomes positive, it corresponds to an unphysical solution because fields begin exponentially growth 
at spatial infinity and near the horizon. Put differently, this situation occurs when the effective potential $V_{s}(r)$ turns out to be negative within the physical domain $\left[r_{+}, \infty\right)$.

Below, we will explore a possibility to find negative branches of the effective potentials for small perturbations over the quasi-spherical neutral $\mathrm{BH}$ background with axial symmetry in Minkowski and AdS spacetimes.

\subsection{Flat Spacetime} $\left.\kappa^{2}=0\right)$

In Minkowski spacetime with $f(r)=1-r_{+} / r$, the effective potential (see (33) for

$$
V_{s}=\frac{f(r)}{r^{2}}\left(\left(1-s^{2}\right) \frac{r_{+}}{r}+v(v+1)\right)
$$

could be negative only for odd (axial) gravitational perturbations with $s=+2$ (clearly, the polar tensor perturbations with the Zerilli potential (29) do not satisfy this condition); it happens for

$$
v(v+1)<\frac{3 r_{+}}{r}, r \in\left[r_{+}, \infty\right) .
$$

For $v^{\prime}$ s satisfying (62), $V_{+2}(r)$ is negative within the interval $r \in\left[r_{+}, r_{0}\right)$ with $r_{0}=$ $\frac{3 r_{+}}{v(v+1)}$. Then, from $r_{0}>r_{+}$, we get the following condition on the separation constant: $v(v+1)<3$, or $v<1.303$. Eigenvalues $v_{2 m}$ smaller than the critical value $v_{c r}=1.303$ produce the negative effective potential. Recall that, in the spherically symmetric case, $v \rightarrow l \geq 2$, so that the separation constant lowest value is $l(l+1)=6$. Hence, the effective potential $V_{s}(r)$ for $s=0,1, \pm 2$ is always positive. According to (59) that gives $\operatorname{Im} \omega<0$ and the standard, Schwarzschild background is stable against small perturbations $[70,71,77]$.

In contrast, small perturbations over a quasi-spherical axially-symmetric $\mathrm{BH}$ background are characterized by three different values of $v_{l m}$ with $l=2$, viz., $v_{20}, v_{21}, v_{22}$. Their dependences on the deformation degree $\alpha$ are depicted in Figure 3 in accordance to the relation (46). One can notice that there are values of $\alpha$ for which $v_{22}$ and $v_{21}$ become smaller of the critical value $v_{c r}=1.303$; hence, we can expect the appearance of QNMs with a positive imaginary part.

To find the QNMs, we follow the semianalytical Padé approximation [88,94,95], improving the standard Wentzel-Kramers-Brillouin (WKB) technique, and the numerical Leaver method [90] based on the continued fraction. As we have discussed, we are mainly interested in values of the QNMs frequencies corresponding to tensor perturbations with eigenvalues $v_{20}, v_{21}, v_{22}$. The eigenvalue $v_{20}$ coincides with that of the spherically symmetric case; therefore, all possible QNMs (fundamental and overtones) related to this case will have the negative imaginary part. The other QNMs for eigenvalues $v_{21}, v_{22}$ (of frequency $\omega_{21}$ and $\omega_{22}$, respectively) are functions of the deformation degree $\alpha$. The corresponding frequencies (actually, their real and imaginary parts) are shown in Figure 6. We find that, for $\alpha>1$, the imaginary part of $\omega_{22}$ becomes greater than zero (see Figure 7). The critical value of the deformation parameter $\alpha=1$ corresponds to $v_{22}=1$ (cf. (46)), so that the domain of negativity of $V_{+2}(r)$ is determined by $\left[r_{+}, \frac{3}{2} r_{+}\right)$. Increasing $\alpha$, this domain increases; $\operatorname{Im} \omega_{22}$ will increase, too. For some values of $\alpha>1$, the imaginary parts of other tensor QNMs also become positive. To sum up, above the critical value of the deformation degree $\alpha=1$, the spacetime geometry of a distorted/quasi-spherical BH becomes unstable against small tensor perturbations. 

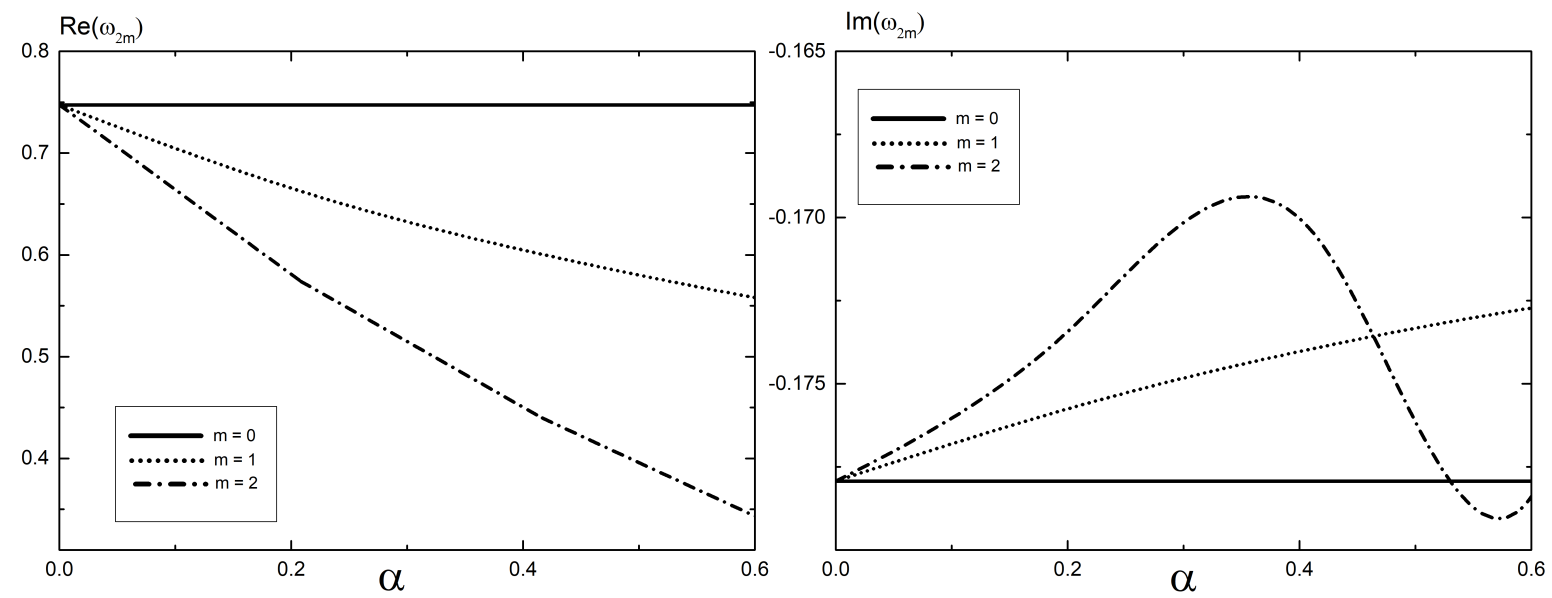

Figure 6. (Left panel) The real part of the lowest odd gravitational perturbations $\omega_{2 m}(\alpha)$ over a quasi-spherical axiallysymmetric neutral BH background in flat spacetime. (Right panel) The imaginary part of $\omega_{2 m}(\alpha)$ under the same conditions.

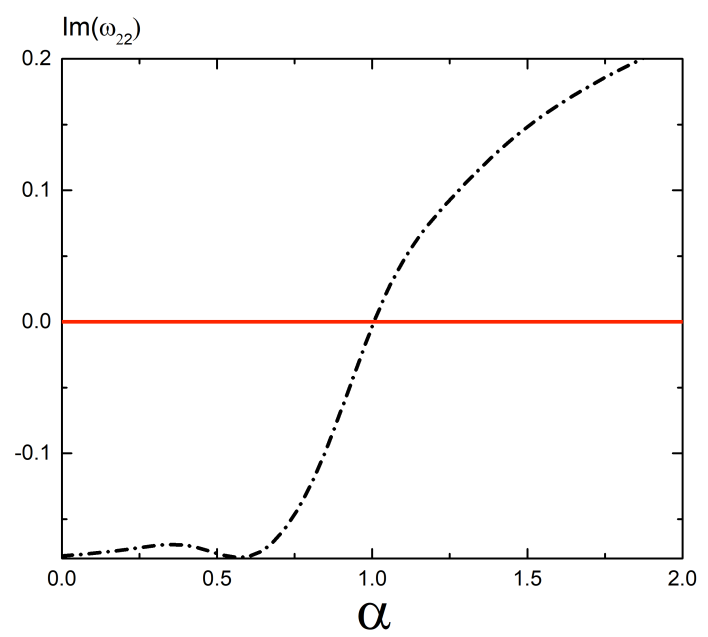

Figure 7. The imaginary part of $\omega_{22}(\alpha)$ in more detail.

\subsection{AdS Spacetime}

Now, turn to AdS spacetime. Here, we have $f(r)=1+r^{2} \kappa^{2}-\frac{r_{+}}{r}\left(1+\kappa^{2} r_{+}^{2}\right)$, and the effective potential of odd perturbations

$$
V_{s}=\frac{f(r)}{r^{2}}\left(\left(1-s^{2}\right)\left(2 \kappa^{2} r+\left(1+\kappa^{2} r_{+}^{2}\right) \frac{r_{+}}{r}\right)+v(v+1)+3 s(s-1) \kappa^{2} r^{2}\right)
$$

could still be negative just for $s=+2$ (the effective potential for even tensor perturbations (29) is positive for any $\left.v_{l m} \geq 2\right)$. The condition of $V_{+2}(r)$ negativity is

$$
v(v+1)<\left(1+\kappa^{2} r_{+}^{2}\right) \frac{3 r_{+}}{r},
$$

and $V_{+2}(r)<0$ within $r \in\left[r_{+}, r_{0}\right)$, where $r_{0}=\frac{3 r_{+}\left(1+\kappa^{2} r_{+}^{2}\right)}{v(v+1)}\left(r_{0}>r_{+}\right)$. Therefore, for eigenvalues $v$, we get

$$
v(v+1)<3\left(1+\kappa^{2} r_{+}^{2}\right) .
$$

Apparently, the result strongly depends on the specific value of $\kappa^{2} r_{+}^{2}\left(r_{+}\right.$is the radius of the event horizon), so now $v$ depends on the size of a BH. In general, the value of $v_{c r}$ in AdS becomes higher than that of Minkowski spacetime; hence, the QNMs with a positive imaginary part may appear for smaller values of $\alpha$. 
To compute the tensor QNMs, here, we will follow the approach of Ref. [79]. In addition, we will take into account the following features of the fundamental (i.e., least damped) QNMs of a Schwarzschild-AdS BH background, marked in Ref. [81]:

- for large (with $r_{+} \mathcal{K} \simeq 100$ ) and intermediate (with $r_{+} \mathcal{K} \simeq 1$ ) BHs the fundamental quasinormal modes are purely imaginary and scale as $\omega / \kappa \simeq\left(r_{+} \kappa\right)^{-1}$;

- for small BHs the fundamental frequencies get non-trivial real and imaginary parts; the latter behaves as $\operatorname{Im} \omega \simeq-r_{+}$. In the limit $r_{+} \rightarrow 0$, the QNMs turn into normal modes of AdS spacetime, determined by $\omega_{l}^{\text {AdS }}=2 n+l+2$.

In the background of a quasi-spherical axisymmetric neutral $\mathrm{BH}$, we have three different tensor QNMs —of frequencies $\omega_{20}, \omega_{21}$ and $\omega_{22}$ - one of which, $\omega_{20}$, coincides with that of the standard spherically-symmetric AdS-Schwarzschild BH. The other two, $\omega_{21}$ and $\omega_{22}$, become functions of the deformation degree $\alpha$. It turns out that, similar to the spherically-symmetric case, frequencies of the fundamental QNMs for large and intermediate BHs come to be purely imaginary. Their functional dependences on $\alpha$ are plotted in Figure 8. Looking at the left panel of Figure 8, one may notice that, for a chosen deformation degree $\alpha$, the least damped QNM corresponds to $m=2$ that gives the smallest value of $v$. Values of the deformation degree $\alpha=0.2$ and $\alpha=0.5$ still give the negative imaginary part of the QNM frequencies. However, increasing the value of $\alpha$ may trigger flipping the sign of $\operatorname{Im} \omega$. Indeed, in the right panel of Figure 8, one finds dependences of $\operatorname{Im} \omega_{21}$ and $\operatorname{Im} \omega_{22}$ for a large BH (with $\kappa r_{+}=10.0$ ) on the parameter $\alpha$. Starting from $\alpha>1, \operatorname{Im} \omega_{22}$ turns out to be positive. It corresponds to $v_{22}<1$. In contrast, $\operatorname{Im} \omega_{21}$ remains negative, even for those $\alpha$ for which $V_{+2}(r)<0$.
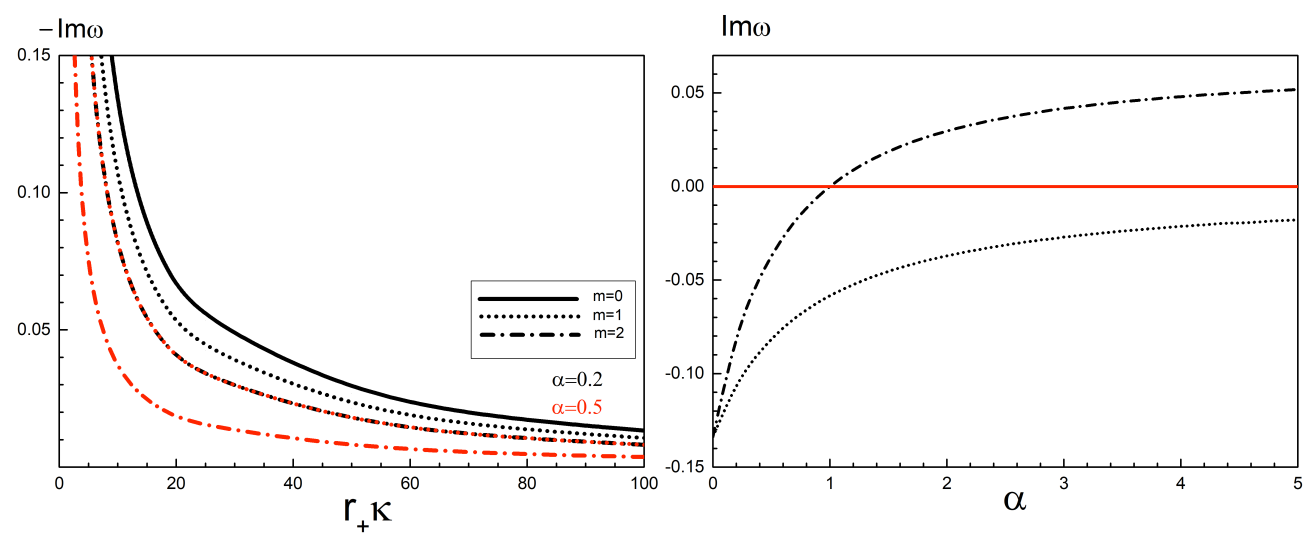

Figure 8. (Left panel) Imaginary parts of $\omega_{2 m}$ as functions of $r_{+} \kappa$. (Right panel) Imaginary parts of Quasinormal modes (QNMs) of frequencies $\omega_{2 m}(m=1,2)$ as functions of the deformation degree $\alpha$.

In addition, in the left panel of Figure 8, we can find that pure imaginary QNMs of large and intermediate BHs depend on $r_{+} \kappa$ quite similarly. This observation allows us to conclude that, for any fixed value of $\alpha$, one can find the spherically symmetric counterpart (of large and intermediate quasi-spherical BHs) with a larger value of the horizon location $r_{+}$, which possesses the same fundamental QNMs. The exception is the $\omega_{22}$ mode, the imaginary part of which becomes positive for $\alpha>1$, so such a correspondence does not take place.

Examining the QNMs of small AdS-Schwarzschild BHs, we find they behave much like the standard spherically-symmetric case. One observes that $\operatorname{Im} \omega \simeq-r_{+}$within the range $r_{+} \mathcal{K} \in[0.3 ; 0,8]$ (right panel of Figure 9). Once $r_{+} \rightarrow 0$, the real parts of the fundamental QNMs are determined by the expression for normal fundamental modes of empty AdS spacetime, $\omega_{\text {AdS }}=l+2$, in which $l$ is replaced with $v$ (left panel of Figure 9).

Therefore, as in the case of flat spacetime, we have established the critical value of the deformation degree $\alpha=1$, below which the background geometry of distorted/quasispherical BHs is stable against small tensor perturbations, but above which the background geometry of large quasi-spherical BHs becomes unstable. 

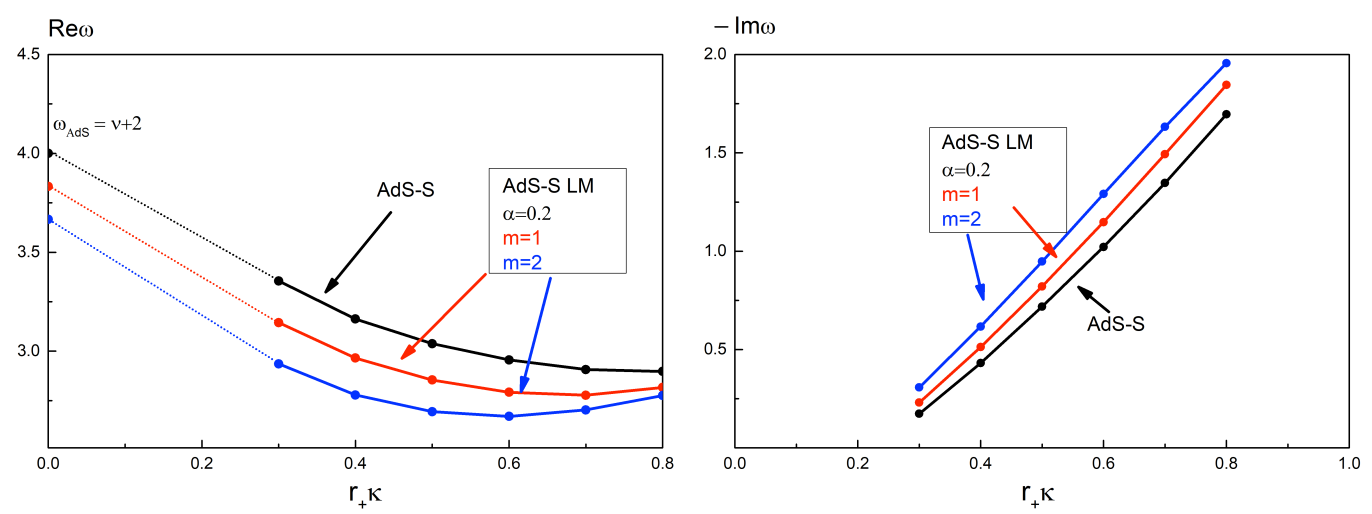

Figure 9. Functional dependences on $r_{+} \kappa$ of real (left panel) and imaginary (right panel) parts of the fundamental QNMs of frequencies $\omega_{2 m}(m=1,2)$. (Small BHs; the deformation degree is fixed to be $\alpha=0.2$.) AdS-S stands for Anti-de Sitter-Schwarzschild; AdS-S LM is the shorthand notation of Anti-de Sitter-Schwarzschild with the Liouville Mode (distorted BH).

\section{Summary and Open Questions}

Let us make a brief sum up of our achievements and findings. We have discussed the scattering problem for small perturbations-scalar, vector, and tensor-on quasi-spherical BHs in Minkowski and AdS spacetimes. Studying the problem, in general, has resulted in the following observations:

- There is a deep connection between distorted and quasi-spherical static BHs. To be precise, we have established the generalization of the Weyl-Erez-Rosen solution to the flatspace Einstein vacuum equations that is reduced to the quasi-spherical Schwarzschild $\mathrm{BH}$ solution, the metric potential of which obeys the Liouville equation.

- The obtained BH spacetime is of type D in the Petrov classification. It makes it possible, despite the spherical symmetry breaking, to separate the variables in dynamical equations of small perturbations and to arrive at the generalized Regge-WheelerZerilli equations, as well as to the generalization of the spherical harmonics equation.

- The outcomes of the spherical symmetry breaking are: non-integer eigenvalues in the generalized spectral problem, coming from the angular part of dynamical equations of small perturbations; their multi-dimensional character for each scattering mode, when the dimension of the appropriate set of eigenvalues is determined by the degree of the corresponding spherical harmonics; and last, the functional dependence of the generalized eigenvalues on the deformation degree parameters.

Restoring a part of the spherical symmetry-the axial symmetry of the spacetime background-relevant for a bunch of astrophysical problems has led us to the following findings:

- The angular dependence of corresponding quantities is further reduced to the dependence on the single polar angle; the spherical Liouville equation for the metric potential turns into the enlargement of general Legendre equation, which can be explicitly solved.

- It turns out that the eigenvalues of the generalized spectral problem depend solely on one parameter of the deformation degree after all. And this functional dependence has been recovered in analytical form. In addition, it has been observed that the generalized eigenvalues are quantized in non-integers.

- For every scattering mode corresponding to the appropriate degree of spherical harmonics $l$, there are $l+1$ different values of the grey-body factors, properties of which are determined by the deformation degree. For instance, we have observed that the grey-body factors increase with increasing the value of the deformation.

- Studying the issue of stability of BH backgrounds, we have found that the value of the deformation degree equal to one is the critical value for the stability of the axially 
symmetric quasi-spherical Schwarzschild BH in Minkowski and AdS spacetimes against the specific small tensor perturbations.

- We also find that, for large and intermediate AdS-Schwarzschild quasi-spherical BHs, the fundamental tensor QNMs of any fixed admissible value of the deformation degree are the same as that of a spherically-symmetric $\mathrm{BH}$ with a larger value of the event horizon along the radial direction.

Therefore, we have observed significant differences in scattering characteristics of gravitational waves caused by losing the spherical symmetry of the background spacetime of their propagation. It would be interesting to find signs of the established effects in the data of real astrophysical observations, at least for slowly rotating systems.

Finally, we will touch upon the following point. It is well known the tidal deformation of compact objects in double neutron star systems is described by the famous I-Love-Q relation; see Refs. [96,97] for reviews. It seems to be important to figure out any possible relation between the effective metric used in Refs. [96,97] and that of the distorted Kerr. Another direction of further studies is related to recovering the metric of a quasi-spherical rotating $\mathrm{BH}$ and studying the scattering processes in a more realistic setup. Our preliminary investigations showed the fail of the Newman-Janis algorithm [98] (also see Refs. [99-103] and Refs. therein) upon constructing the rotating extension of a quasi-spherical static metric, mostly used in the paper. Perhaps, the observed here connection between distorted and quasi-spherical BHs will make possible to complete this task in another way. We hope to continue studies on this and other related topics and report results in forthcoming publications.

Author Contributions: Conceptualization and methodology, A.J.N.; investigation, A.M.A., A.J.N.; writing—original draft preparation, A.M.A., A.J.N.; writing—review and editing, A.J.N.; supervision, A.J.N. All authors have read and agreed to the published version of the manuscript.

Funding: This research received no external funding.

Acknowledgments: A.M.A. is grateful to the Institute for Theoretical Physics of NSC KIPT, where part of this work was completed, for its warm hospitality. A.J.N. acknowledges all the colleagues, with whome the subject of the present research was discussed, for fruiful and stimulating conversations. The authors are thankful to the anonymous reviewers for their suggestions in improving the early version of the paper.

Conflicts of Interest: The authors declare no conflict of interest.

\section{Abbreviations}

The following abbreviations are used in this manuscript:

$\begin{array}{ll}\text { AdS } & \text { anti-de Sitter } \\ \text { AGN } & \text { active galactic nuclei } \\ \text { BH } & \text { black hole } \\ \text { EHT } & \text { event horizon telescope } \\ \text { GBF } & \text { grey-body factor } \\ \text { GR } & \text { general relativity } \\ \text { GW } & \text { gravitational wave } \\ \text { NP } & \text { Newman-Penrose } \\ \text { QNM } & \text { quasinormal mode } \\ \text { UHECRs } & \text { ultra high energy cosmic rays } \\ \text { SMBH } & \text { super massive black hole } \\ \text { WKB } & \text { Wentzel-Kramers-Brillouin }\end{array}$

\section{Appendix A. Spherically-Symmetric and Axially-Symmetric Solutions to the Liouville Equation}

First, let us briefly discuss the way to solve the spherical Liouville Equation (13) in terms of unconstrained functions. See Appendix B of Ref. [64] for more details. 
To this end, it is convenient to turn to the stereographic projection plane coordinates

$$
z=e^{i \varphi} \tan \frac{\theta}{2}, \quad \bar{z}=e^{-i \varphi} \tan \frac{\theta}{2} .
$$

Then, the angular part of $S^{2}$ line element, $d s^{2}=d \theta^{2}+\sin ^{2} \theta d \varphi^{2}$, becomes the FubiniStudy metric of $C P^{1}$ complex projective space:

$$
d s^{2}=\frac{4}{(1+z \bar{z})^{2}} d z d \bar{z},
$$

and the angular part of the Laplacian (14) is simplified up to

$$
\Delta_{\theta, \varphi}=\frac{1}{4}(1+z \bar{z})^{2} \partial_{z} \partial_{\bar{z}} .
$$

Consequently, the spherical Liouville Equation (13) for $\chi(\theta, \varphi)$ takes the form of

$$
\frac{1}{4}(1+z \bar{z})^{2} \partial_{z} \partial_{\bar{z}} \chi(z, \bar{z})+2 e^{\chi(z, \bar{z})}-2=0
$$

and can be solved in terms of arbitrary complex analytical function $F(z)[63,64]$ :

$$
\chi(z, \bar{z})=-2 \ln [F(z) \bar{F}(\bar{z})+1]+\ln \left[\frac{d F(z)}{d z} \frac{d \bar{F}(\bar{z})}{d \bar{z}}\right]+\ln (1+z \bar{z})^{2} .
$$

Second, let us survey the way of getting expression (40) in the case of axial symmetry of a quasi-spherical BH. Turning to the isothermal coordinates $(x, y)$ in $z$, viz. $z=x+i y$, for (A4), we obtain

$$
\frac{1}{4}\left(1+x^{2}+y^{2}\right)^{2}\left(\frac{\partial^{2} \chi}{\partial x^{2}}+\frac{\partial^{2} \chi}{\partial y^{2}}\right)+2 e^{\chi(x, y)}-2=0 .
$$

The Fubini-Study metric (A3) comes to

$$
d s^{2}=e^{\chi_{0}(x, y)}\left(d x^{2}+d y^{2}\right),
$$

with

$$
e^{\chi_{0}(x, y)} \equiv \frac{4}{\left(1+x^{2}+y^{2}\right)^{2}}
$$

or

$$
\chi_{0}(x, y)=2 \ln \left[\frac{2}{1+x^{2}+y^{2}}\right] \text {. }
$$

Next, we define a function $\Phi(x, y)$ as

$$
\chi(x, y)=\Phi(x, y)-\chi_{0}(x, y)=\Phi(x, y)-2 \ln \left[\frac{2}{1+x^{2}+y^{2}}\right] .
$$

Substituting (A10) into (A6) leads to the following equation for $\Phi(x, y)$ :

$$
\frac{1}{4}\left(1+x^{2}+y^{2}\right)^{2}\left[\frac{\partial^{2} \Phi}{\partial x^{2}}+\frac{\partial^{2} \Phi}{\partial y^{2}}+2 e^{\Phi(x, y)}\right]=0 \leadsto \frac{\partial^{2} \Phi}{\partial x^{2}}+\frac{\partial^{2} \Phi}{\partial y^{2}}+2 e^{\Phi(x, y)}=0 .
$$

Now, the axially-symmetric case corresponds to demanding $\Phi(x, y)$ to solely depend on the radial coordinate $\rho$, related to $(x, y)$ via $\rho^{2}=x^{2}+y^{2}$. Hence, $\Phi(x, y) \rightarrow \Phi(\rho)$, and $\Phi(\rho)$ obeys

$$
\frac{d^{2} \Phi(\rho)}{d \rho^{2}}+\frac{1}{\rho} \frac{d \Phi(\rho)}{d \rho}+2 e^{\Phi(\rho)}=0 .
$$


With $\Phi=\ln f(\rho)$, Equation (A12) transforms into

$$
f \frac{d^{2} f(\rho)}{d \rho^{2}}-\left(\frac{d f(\rho)}{d \rho}\right)^{2}+\frac{f}{\rho} \frac{d f(\rho)}{\rho}+2 f^{3}(\rho)=0,
$$

the solution to which is

$$
f(\rho)=\frac{2+C_{1}}{2 \rho^{2} \cosh ^{2}\left(\frac{\sqrt{2+C_{1}}\left(C_{2}-\ln \rho\right)}{\sqrt{2}}\right)} .
$$

Turning to Equation (A10) back, for $\chi(\rho)$, we have

$$
\chi(\rho)=\ln \left[\frac{\left(2+C_{1}\right)\left(1+\rho^{2}\right)^{2}}{8 \rho^{2} \cosh ^{2}\left(\frac{\sqrt{2+C_{1}}\left(C_{2}-\ln \rho\right)}{\sqrt{2}}\right)}\right] .
$$

Finally, with inserting new constants $a=\sqrt{1+\frac{C_{1}}{2}}, b=e^{a C_{2}}$ and replacing $\rho$ with its functional dependence on $\theta, \rho=\tan \frac{\theta}{2}$ (cf. (A1)), we arrive at

$$
e^{\chi(\theta)}=\left(\frac{a}{b}\right)^{2} \tan ^{2 a-2} \frac{\theta}{2}\left(\frac{1+\tan ^{2} \frac{\theta}{2}}{\left(1+b^{-2} \tan ^{2 a} \frac{\theta}{2}\right)}\right)^{2} .
$$

On account of the trigonometric identity $\tan ^{2} \theta / 2=(1-x) /(1+x), x=\cos \theta$, the obtained solution (A16) to the Liouville equation in polar coordinates turns into expression (40).

\section{References}

1. Bartos, I.; Kowalski, M. Multimessenger Astronomy; IOP Publishing: Bristol, UK, 2017. [CrossRef]

2. Abbott, B.P.; LIGO Scientific Collaboration; Virgo Collaboration. Binary Black Hole Mergers in the first Advanced LIGO Observing Run. Phys. Rev. X 2016, 6, 041015; Erratum in 2018, 8, 039903. [CrossRef]

3. Pierre Auger Collaboration. Correlation of the highest energy cosmic rays with nearby extragalactic objects. Science 2007, 318,938 . [CrossRef] [PubMed]

4. Eckart, A.; Genzel, R. Observations of stellar proper motions near the Galactic Centre. Nature 1996, 383, 415. [CrossRef]

5. Ghez, A.M.; Klein, B.L.; Morris, M.; Becklin, E.E. High proper motion stars in the vicinity of Sgr A*: Evidence for a supermassive black hole at the center of our galaxy. Astrophys. J. 1998, 509, 678. [CrossRef]

6. De Felice, F.; Sorge, F. Magnetized orbits around a Schwarzschild black hole. Class. Quant. Grav. 2003, 20, 469. [CrossRef]

7. De Felice, F.; Sorge, F.; Zilio, S. Magnetized orbits around a Kerr black hole. Class. Quant. Grav. 2004, 21, 961. [CrossRef]

8. Kachelriess, M. Lecture notes on high energy cosmic rays. arXiv 2008, arXiv:0801.4376.

9. Stanev, T. High Energy Cosmic Rays; Springer \& Praxis: Berlin, Germany; Chichester, UK, 2010. [CrossRef]

10. Dawson, B.R.; Fukushima, M.; Sokolsky, P. Past, Present and Future of UHECR Observations. PTEP 2017, 12, 12A101. [CrossRef]

11. Park, I.Y. Quantum-corrected Geometry of Horizon Vicinity. Fortsch. Phys. 2017, 65, 1700038. [CrossRef]

12. Nurmagambetov, A.J.; Park, I.Y. Quantum-induced trans-Planckian energy near horizon JHEP 2018, 1805, 167. [CrossRef]

13. Guépin, C.; Kotera, K.; Barausse, E.; Fang, K.; Murase, K. Ultra-High Energy Cosmic Rays and Neutrinos from Tidal Disruptions by Massive Black Holes. Astron. Astrophys. 2018, 616, A179; Erratum in 2020, 636, C3. [CrossRef]

14. Nurmagambetov, A.J.; Park, I.Y. Quantum-gravitational trans-Planckian energy of a time-dependent black hole. Symmetry 2019, 11, 1303. [CrossRef]

15. Nurmagambetov, A.J.; Park, I.Y. On Firewalls in quantum-corrected General Relativity. J. Phys. Conf. Ser. 2019, $1390,012091$. [CrossRef]

16. Comisso, L.; Sironi, L. The interplay of magnetically-dominated turbulence and magnetic reconnection in producing nonthermal particles. Astrophys. J. 2019, 886, 122. [CrossRef]

17. Nurmagambetov, A.J. Quantum Leaps in the Vicinity of One-Loop Gravity Black Holes. Phys. Part. Nucl. 2020, 51, 739. [CrossRef]

18. Nurmagambetov, A.J.; Park, I.Y. Quantum-gravitational trans-Planckian radiation by a rotating black hole. arXiv 2020, arXiv:2007.06070.

19. Comisso, L.; Asenjo, F.A. Magnetic Reconnection as a Mechanism for Energy Extraction from Rotating Black Holes. arXiv 2020, arXiv:2012.00879.

20. Event Horizon Telescope Collaboration. First M87 Event Horizon Telescope Results. I. The Shadow of the Supermassive Black Hole. Astrophys. J. 2019, 875, L1. [CrossRef] 
21. EHT Collaboration. Gravitational Test beyond the First Post-Newtonian Order with the Shadow of the M87 Black Hole. Phys. Rev. Lett. 2020, 125, 141104. [CrossRef]

22. Gralla, S.E. Can the EHT M87 results be used to test general relativity? arXiv 2020, arXiv:2010.08557.

23. Starobinskii, A.A.; Churilov, S.M. Amplification of electromagnetic and gravitational waves scattered by a rotating "black hole". Sov. Phys. JETP 1974, 38, 1-5.

24. Fabbri, R. Scattering and absorption of electromagnetic waves by a Schwarzschild black hole. Phys. Rev. D 1975, 12, 933-942. [CrossRef]

25. Unruh, W.G. Absorption Cross-Section of Small Black Holes. Phys. Rev. D 1976, 14, 3251-3259. [CrossRef]

26. Sanchez, N.G. Scattering of scalar waves from a Schwarzschild black hole. J. Math. Phys. 1976, 17, 688-692. [CrossRef]

27. Sanchez, N.G. The Wave Scattering Theory and the Absorption Problem for a Black Hole. Phys. Rev. D 1977, 16, 937-945. [CrossRef]

28. Sanchez, N.G. Absorption and Emission Spectra of a Schwarzschild Black Hole. Phys. Rev. D 1978, 18, 1030-1036. [CrossRef]

29. Sanchez, N.G. Elastic Scattering of Waves by a Black Hole. Phys. Rev. D 1978, 18, 1798-1804. [CrossRef]

30. Cvetic, M.; Larsen, F. Grey body factors for rotating black holes in four-dimensions. Nucl. Phys. B 1997, 506, 107-120. [CrossRef]

31. Klebanov, I.R.; Mathur, S.D. Black hole grey body factors and absorption of scalars by effective strings. Nucl. Phys. B 1997, 500, 115-132. [CrossRef]

32. Cvetic, M.; Larsen, F. Greybody factors for black holes in four-dimensions: Particles with spin. Phys. Rev. D 1998, 57, 6297-6310. [CrossRef]

33. Kanti, P. Black holes in theories with large extra dimensions: A Review. Int. J. Mod. Phys. A 2004, 19, 4899-4951. [CrossRef]

34. Grain, J.; Barrau, A.; Kanti, P. Exact results for evaporating black holes in curvature-squared lovelock gravity: Gauss-Bonnet greybody factors. Phys. Rev. D 2005, 72, 104016. [CrossRef]

35. Keshet, U.; Neitzke, A. Asymptotic spectroscopy of rotating black holes. Phys. Rev. D 2008, 78, 044006. [CrossRef]

36. Boonserm, P.; Visser, M. Bounding the greybody factors for Schwarzschild black holes. Phys. Rev. D 2008, 78, 101502. [CrossRef]

37. Li, W.; Xu, L.; Liu, M. Greybody factors in rotating charged Goedel black holes. Class. Quant. Grav. 2009, 26, 055008. [CrossRef]

38. Harmark, T.; Natario J.; Schiappa R. Greybody Factors for d-Dimensional Black Holes. Adv. Theor. Math. Phys. 2010, 14, 727-793. [CrossRef]

39. Gonzalez, P.; Papantonopoulos, E.; Saavedra, J. Chern-Simons black holes: Scalar perturbations, mass and area spectrum and greybody factors. JHEP 2010, 8, 50. [CrossRef]

40. Boonserm, P.; Ngampitipan, T.; Visser, M. Regge-Wheeler equation, linear stability, and greybody factors for dirty black holes. Phys. Rev. D 2013, 88, 041502. [CrossRef]

41. Dong, R.; Stojkovic, D. Greybody factors for a black hole in massive gravity. Phys. Rev. D 2015, 92, 084045. [CrossRef]

42. Catalán, M.; Cisternas, E.; González, P.A.; Vásquez, Y. Quasinormal modes and greybody factors of a four-dimensional Lifshitz black hole with z = 0. Astrophys. Space Sci. 2016, 361, 189. [CrossRef]

43. Gray, F.; Visser, M. Greybody Factors for Schwarzschild Black Holes: Path-Ordered Exponentials and Product Integrals. Universe 2018, 4, 93. [CrossRef]

44. Stephani, H.; Kramer, D.; MacCallum, M.; Hoenselaers, C.; Herlt, E. Exact Solutions of Einstein's Field Equations; Cambridge University Press: Cambridge, UK, 2003. [CrossRef]

45. Griffiths, J.B.; Podolsky, J. Exact Space-Times in Einstein's General Relativity; Cambridge University Press: Cambridge, UK, 2009.

46. Weyl, H. Zur Gravitationstheorie. Ann. Phys. 1917, 54, 117-145. [CrossRef]

47. Geroch, R.; Hartle, J.B. Distorted black holes. J. Math. Phys. 1982, 23, 680-692. [CrossRef]

48. Thorne, K.S. Nonspherical Gravitational Collapse: Does it Produce Black Holes? Comments Astrophys. Space Phys. 1970, 2, 191-196.

49. Thorne, K.S. Nonspherical Gravitational Collapse: A Short Review. In Magic without Magic; Klauder, J.R., Ed.; Freeman: San Francisco, CA, USA,1972; pp. 231-258.

50. Tomimatsu, A. Distorted Rotating Black Holes. Phys. Lett. A 1984, 103, 374-376. [CrossRef]

51. Breton, N.; Denisova, T.E.; Manko, V.S. A Kerr black hole in the external gravitational field. Phys. Lett. A 1997, $230,7-11$. [CrossRef]

52. Breton, N.; Garcia, A.A.; Manko, V.S.; Denisova, T.E. Arbitrarily deformed Kerr Newman black hole in an external gravitational field. Phys. Rev. D 1998, 57, 3382-3388. [CrossRef]

53. Semerák, O.; Žačék, M. Gravitating discs around a Schwarzschild black hole: I. Class. Quant. Grav. 2000, 17, 1613-1626. [CrossRef]

54. Žačék, M.; Semerák, O. Gravitating discs around a Schwarzschild black hole: II. Czech. J. Phys. 2002, 52, 19-27. [CrossRef]

55. Letelier, P.S. On the stability of circular orbits of particles moving around black holes surrounded by axially symmetric structures. Phys. Rev. D 2003, 68, 104002. [CrossRef]

56. Shoom, A.A.; Walsh, C.; Booth, I. Geodesic motion around a distorted static black hole. Phys. Rev. D 2016, 93, 064019. [CrossRef]

57. Kunz, J.; Nedkova, P.; Yazadjiev, S. Magnetized Black Holes in an External Gravitational Field. Phys. Rev. D 2017, $96,024017$. [CrossRef]

58. Araujo, M.E.; Oliveira, S.R. Static axisymmetric approach for the headon collision of two black holes. Phys. Rev. D 1995, 52, 816-820. [CrossRef]

59. Araujo, M.E.; Letelier, P.S.; Oliveira, S.R. Two Kerr black holes with axisymmetric spins: An Improved Newtonian model for the head-On collision and gravitational radiation. Class. Quant. Grav. 1998, 15, 3051-3060. [CrossRef] 
60. Semerák, O.; Basovník, M. Geometry of deformed black holes. I. Majumdar-Papapetrou binary. Phys. Rev. D 2016, $94,044006$. [CrossRef]

61. Moskalets, T.; Nurmagambetov, A. Liouville mode in Gauge/Gravity Duality. Eur. Phys. J. C 2015, 75, 551. [CrossRef]

62. Moskalets, T.M.; Nurmagambetov A.J. Non-uniform horizons in Gauge/Gravity Duality. Phys. Atom. Nucl. 2016, 79, 1497-1499. [CrossRef]

63. Moskalets, T.M.; Nurmagambetov A.J. Static and non-static black holes with the Liouville mode. Phys. Part. Nucl. Lett. 2017, 14, 365-367. [CrossRef]

64. Moskalets, T.; Nurmagambetov, A. Absorption cross-sections of small quasi-spherical black holes: The massless scalar case. arXiv 2016, arXiv:1607.08830.

65. Boos, J.; Frolov, V.P. Stationary black holes with stringy hair. Phys. Rev. D 2018, 97, 024024. [CrossRef]

66. Erez, G.; Rosen, N. The Gravitational Field of a Particle Possessing a Multipole Moment. Bull. Res. Counc. Isr. 1959, 8F, 47-50.

67. Quevedo, H. General static axisymmetric solution of Einstein's vacuum field equations in prolate spheroidal coordinates. Phys. Rev. D 1989, 39, 2904-2911. [CrossRef] [PubMed]

68. Crowdy, D.G. General solutions to the 2D Liouville equation. Int. J. Eng. Sci. 1997, 35, 141. [CrossRef]

69. Popov, A.G. Exact formulae of constructing solutions to the Liouville equation by use of solutions to the Laplace equation. Dokl. Akad. Nauk 1993, 333, 440-441. (In Russian)

70. Regge, T.; Wheeler J.A. Stability of a Schwarzschild singularity. Phys. Rev. 1957, 108, 1063-1069. [CrossRef]

71. Zerilli, F.J. Effective potential for even parity Regge-Wheeler gravitational perturbation equations. Phys. Rev. Lett. 1970, 24, 737-738. [CrossRef]

72. Petrov, A.Z. The classification of spaces defining gravitational fields. Sci. Not. Kazan State Univ. 1954, 114, 55-69. [CrossRef]

73. Petrov, A.Z. Einstein Spaces; Pergamon Press: Oxford, UK, 1969.

74. Newman, E.; Penrose R. An Approach to gravitational radiation by a method of spin coefficients. J. Math. Phys. 1961, 3, 566-578. [CrossRef]

75. Teukolsky, S.A. Perturbations of a rotating black hole. 1. Fundamental equations for gravitational electromagnetic and neutrino field perturbations. Astrophys. J. 1973, 185, 635-647. [CrossRef]

76. Press, W.H.; Teukolsky S.A. Perturbations of a Rotating Black Hole. II. Dynamical Stability of the Kerr Metric. Astrophys. J. 1973, 185, 649-674. [CrossRef]

77. Chandrasekhar, S. The Mathematical Theory of Black Holes; Oxford University Press: Oxford, UK, 1983.

78. Otsuki, H.; Futamase, T. Gravitational Perturbation of Schwarzschild-De Sitter Spacetime and Its Quasi-Normal Modes. Prog. Theor. Phys. 1991, 85, 771-778. [CrossRef]

79. Horowitz, G.T.; Hubeny, V.E. Quasinormal modes of AdS black holes and the approach to thermal equilibrium. Phys. Rev. D 2000, 62, 024027. [CrossRef]

80. Cardoso, V.; Lemos, J.P.S. Quasinormal modes of Schwarzschild anti-de Sitter black holes: Electromagnetic and gravitational perturbations. Phys. Rev. D 2001, 64, 084017. [CrossRef]

81. Cardoso, V.; Konoplya, R.; Lemos, J.P.S. Quasinormal frequencies of Schwarzschild black holes in anti-de Sitter space-times: A Complete study on the asymptotic behavior. Phys. Rev. D 2003, 68, 044024. [CrossRef]

82. Burgess, C.P.; Lutken, C.A. Propagators and Effective Potentials in Anti-de Sitter Space. Phys. Lett. B 1985, 153, $137-141$. [CrossRef]

83. Konoplya, R.A.; Zhidenko, A. Quasinormal modes of black holes: From astrophysics to string theory. Rev. Mod. Phys. 2011, 83, 793-836. [CrossRef]

84. Dias, O.J.C.; Reall, H.S.; Santos, J.E. Strong cosmic censorship: Taking the rough with the smooth. JHEP 2018, 10, 001. [CrossRef]

85. Glampedakis, K.; Johnson, A.D.; Kennefick, D. Darboux transformation in black hole perturbation theory. Phys. Rev. D 2017, 96, 024036. [CrossRef]

86. Moulin, F.; Barrau, A. Analytical proof of the isospectrality of quasinormal modes for Schwarzschild-de Sitter and SchwarzschildAnti de Sitter spacetimes. Gen. Rel. Grav. 2020, 52, 82. [CrossRef]

87. Arslanaliev, A.M.; Nurmagambetov, A.J. Price's Theorem in Gauge/Gravity Duality. Phys. Part. Nucl. 2018, 49, 879-883. [CrossRef]

88. Konoplya, R.A. Quasinormal behavior of the d-dimensional Schwarzschild black hole and higher order WKB approach. Phys. Rev. D 2003, 68, 024018. [CrossRef]

89. Lin, K.; Qian, W.L. A Matrix Method for Quasinormal Modes: Schwarzschild Black Holes in Asymptotically Flat and (Anti-) de Sitter Spacetimes. Class. Quant. Grav. 2017, 34, 095004. [CrossRef]

90. Leaver, E.W. An Analytic representation for the quasi normal modes of Kerr black holes. Proc. R. Soc. Lond. A 1985, 402, 285-298.

91. Nollert, H.P. Quasinormal modes: The characteristic 'sound' of black holes and neutron stars. Class. Quant. Grav. 1999, 16, R159-R216. [CrossRef]

92. Berti, E.; Kokkotas, K.D. Quasinormal modes of Reissner-Nordstrom-anti-de Sitter black holes: Scalar, electromagnetic and gravitational perturbations. Phys. Rev. D 2003, 67, 064020. [CrossRef]

93. Ferrari, V.; Gualtieri, L. Quasi-Normal Modes and Gravitational Wave Astronomy. Gen. Rel. Grav. 2008, 40, 945-970. [CrossRef]

94. Matyjasek, J.; Opala M. Quasinormal modes of black holes. The improved semianalytic approach. Phys. Rev. D 2017, 96, 024011. [CrossRef] 
95. Konoplya, R.A.; Zhidenko, A.; Zinhailo, A.F. Higher order WKB formula for quasinormal modes and grey-body factors: Recipes for quick and accurate calculations. Class. Quant. Grav. 2019, 36, 155002. [CrossRef]

96. Yagi, K.; Yunes, N. I-Love-Q Relations in Neutron Stars and their Applications to Astrophysics, Gravitational Waves and Fundamental Physics. Phys. Rev. D 2013, 88, 023009. [CrossRef]

97. Yagi, K.; Yunes, N. I-Love-Q Relations: From Compact Stars to Black Holes. Class. Quant. Grav. 2016, 33, 095005. [CrossRef]

98. Newman, E.T.; Janis, A.I. Note on the Kerr spinning particle metric. J. Math. Phys. 1965, 6, 915-917. [CrossRef]

99. Drake, S.P.; Szekeres, P. Uniqueness of the Newman-Janis algorithm in generating the Kerr-Newman metric. Gen. Rel. Grav. 2000, 32, 445-458. [CrossRef]

100. Ferraro, R. Untangling the Newman-Janis algorithm. Gen. Rel. Grav. 2014, 46, 1705. [CrossRef]

101. Erbin, H. Janis-Newman algorithm: Simplifications and gauge field transformation. Gen. Rel. Grav. 2015, 47, 19. [CrossRef]

102. Rajan, D. Complex Spacetimes and the Newman-Janis trick. arXiv 2016, arXiv:1601.03862.

103. Erbin, H. Janis-Newman algorithm: Generating rotating and NUT charged black holes. Universe 2017, 3, 19. [CrossRef] 